\title{
Numerical analysis of the Oseen-type Peterlin viscoelastic model by the stabilized Lagrange-Galerkin method Part II: A linear scheme
}

\author{
Mária Lukáčová-Medvid'ová ${ }^{1}$, Hana Mizerová ${ }^{1}$, \\ Hirofumi Notsu ${ }^{2,3}$ and Masahisa Tabata ${ }^{4}$ \\ ${ }^{1}$ Institute of Mathematics, University of Mainz, Mainz 55099, Germany \\ ${ }^{2}$ Faculty of Mathematics and Physics, Kanazawa University, Kanazawa 920-1192, Japan \\ ${ }^{3}$ Japan Science and Technology Agency (JST), PRESTO, Saitama 332-0012, Japan \\ ${ }^{4}$ Department of Mathematics, Waseda University, Tokyo 169-8555, Japan
}

\begin{abstract}
This is the second part of our error analysis of the stabilized Lagrange-Galerkin scheme applied to the Oseen-type Peterlin viscoelastic model. Our scheme is a combination of the method of characteristics and BrezziPitkäranta's stabilization method for the conforming linear elements, which leads to an efficient computation with a small number of degrees of freedom especially in three space dimensions. In this paper, Part II, we apply a semi-implicit time discretization which yields the linear scheme. We concentrate on the diffusive viscoelastic model, i.e. in the constitutive equation for time evolution of the conformation tensor a diffusive effect is included. Under mild stability conditions we obtain error estimates with the optimal convergence order for the velocity, pressure and conformation tensor in two and three space dimensions. The theoretical convergence orders are confirmed by numerical experiments.

Keywords: Error estimates, The Peterlin viscoelastic model, Lagrange-Galerkin method, Pressure-stabilization 2010 MSC: 65M12, 76A05, 65M60, 65M25
\end{abstract}

\section{Introduction}

The present paper is a continuation of numerical error analysis of the stabilized Lagrange-Galerkin method applied to the Oseen-type Peterlin viscoelastic model. In our previous paper [29], Part I, we dealt with the fully nonlinear implicit scheme, whereas here, in Part II, we investigate a linear semi-implicit scheme.

The development of stable and convergent numerical methods for viscoelastic models, such as the Oldroyd-B type models, is an active research area. In particular, the question of stability when elastic effects are dominant (the so-called high Weissenberg number problem) remains an open problem. We refer the reader to works of Fattal and Kupferman [20,21], where an interesting approach using the log-conformation representation has been introduced. Furthermore, in Boyaval et al. [10] free energy dissipative Lagrange-Galerkin schemes with or without the log-conformation representation has been studied and in Lee and Xu [27] and Lee et al. [28] finite element schemes using the idea of the generalized Lie derivative have been proposed. Further related numerical schemes and computations can be found, e.g., in $[1,2,7,8,15,26,32,34,36,41,42,53,54]$, see also references therein. To the best of our knowledge there are no results on error estimates of numerical schemes for the Oldroyd-B model, see Picasso and Rappaz [44] and Bonito et al. [6] for error analysis of simplified models without convective terms.

In [43] Peterlin proposed a mean-field closure model according to which the average of the elastic force over thermal fluctuations is replaced by the value of the force at the mean-squared polymer extension. This means that a nonlinear spring force law $F(R)=\gamma\left(|R|^{2}\right) R$ that acts in a dumbbell is replaced by the function $F(R)=\gamma(\operatorname{tr} \mathbf{C}) R$. Here, $\gamma$ is the spring constant, $\mathbf{C}$ is the so-called conformation tensor and $R$ is the vector connecting the beads of a dumbbell. Based on this approach Renardy has recently derived a new class of general macroscopic constitutive models, that is motivated by Peterlin dumbbell theories with a nonlinear spring law for an infinitely extensible spring, see Renardy $[48,49]$ and recent papers by Lukáčová-Medvid'ová et al. [30,31], where the global existence of weak solutions has been obtained. 
In this paper, Part II, as well as in our previous paper, Part I, we consider the so-called Oseen-type Peterlin viscoelastic model that is a system of the flow equations and an equation for the conformation tensor, cf. [47-49]. We concentrate on the diffusive viscoelastic model, which means that in the constitutive equations for the conformation tensor a diffusive effect is included.

Let us point out that in standard derivations of bead-spring models the diffusive term in the equation for the elastic stress tensor is routinely omitted. In [17] a careful justification of the presence of the diffusive term in the Fokker-Planck equations through the asymptotic analysis is presented. The diffusion coefficient $\varepsilon$ is proportional to $(\ell / L)^{2} / W e$, where $L$ and $\ell$ are characteristic macroscopic and microscopic length scales, respectively, and $W e$ is the so-called Weissenberg number. It is a reference number characterizing viscoelastic property of the material. Estimates for $(\ell / L)^{2}$ presented in [5] show that $(\ell / L)^{2}$ is in the range of about $10^{-9}$ and $10^{-7}$. As emphasized in [4] the model reduction by neglecting this small diffusive effect is mathematically counterproductive leading to a degenerate parabolic-hyperbolic system (1) with $\varepsilon=0$. On the other hand, when the diffusive term is taken into account, the resulting system (1) remains parabolic. We would like to point out that in the analysis presented below we only require $\varepsilon>0$ and there is no assumption on the size of $\varepsilon$. For the details of the derivation of the diffusive Peterlin model we refer to $[30,35,48,49]$. Let us mention that, even when the velocity field is given, the equation for the conformation tensor in the Peterlin model is still nonlinear, while the Oldroyd-B model is linear with respect to the extra stress tensor. Hence, we can say that the nonlinearity of the Peterlin model is stronger than that of the Oldroyd-B model. As a starting point of the numerical analysis of the Peterlin model, we consider the Oseen-type model, where the velocity of the material derivative is replaced by a known one, in order to concentrate on the treatment of nonlinear terms arising from the elastic stress.

In the present paper a stabilized Lagrange-Galerkin method for the Peterlin viscoelastic model is studied. It consists of the method of characteristics and Brezzi-Pitkäranta's stabilization method [13] for the conforming linear elements. The method of characteristics derives the robustness in convection-dominated flow problems, and the stabilization method reduces the number of degrees of freedom in computation especially in three space dimensions. In our recent works by Notsu and Tabata [37-39] the stabilized Lagrange-Galerkin method has been applied successfully for the Oseen, Navier-Stokes and natural convection problems and optimal error estimates have been proved. We extend the numerical analysis of the stabilized Lagrange-Galerkin method to the Oseentype Peterlin model. As already mentioned above, the aim of the present paper paper is to give a rigorous error analysis of the linear stabilized Lagrange-Galerkin scheme for the diffusive Peterlin model in both two and three space dimensions. We show that under mild stability conditions the obtained error estimates have the optimal convergence rate.

As mentioned in Boyaval et al. [10], the positive definiteness of the conformation tensor is important in the analysis of numerical schemes for the viscoelastic models, where this property has been shown for the exact strong solution in [35]. We remark that our error estimates have been obtained successfully without studying positive definiteness of the conformation tensor. Let us additionally note that this paper includes the error estimate for the pressure in the standard $L^{2}$ norm (Theorem 2), which has, as far as we know, never been shown for time-dependent viscoelastic flow problems, e.g., the Oldroyd-B model.

This paper is organized as follows. In Section 2 the mathematical model for the Peterlin viscoelastic fluid is described. In Section 3 a linear stabilized Lagrange-Galerkin scheme is presented. The main results on the convergence with optimal error estimates are stated in Section 4, and proved in Section 5 . In Section 6 some numerical experiments confirming the theoretical convergence orders are provided.

\section{The Oseen-type Peterlin viscoelastic model}

The function spaces and the notation to be used throughout the paper are as follows. Let $\Omega$ be a bounded domain in $\mathbb{R}^{d}$ for $d=2$ or $3, \Gamma:=\partial \Omega$ the boundary of $\Omega$, and $T$ a positive constant. For $m \in \mathbb{N} \cup\{0\}$ and $p \in[1, \infty]$ we use the Sobolev spaces $W^{m, p}(\Omega), W_{0}^{1, \infty}(\Omega), H^{m}(\Omega)\left(=W^{m, 2}(\Omega)\right), H_{0}^{1}(\Omega)$ and $L_{0}^{2}(\Omega):=\{q \in$ $\left.L^{2}(\Omega) ; \int_{\Omega} q d x=0\right\}$. Furthermore, we employ function spaces $H_{\text {sym }}^{m}(\Omega):=\left\{\mathbf{D} \in H^{m}(\Omega)^{d \times d} ; \mathbf{D}=\mathbf{D}^{T}\right\}$ and $C_{\text {sym }}^{m}(\bar{\Omega}):=C^{m}(\bar{\Omega})^{d \times d} \cap H_{\text {sym }}^{m}(\Omega)$, where the superscript $T$ stands for the transposition. For any normed space $S$ with norm $\|\cdot\|_{S}$, we define function spaces $H^{m}(0, T ; S)$ and $C([0, T] ; S)$ consisting of $S$-valued functions in $H^{m}(0, T)$ and $C([0, T])$, respectively. We use the same notation $(\cdot, \cdot)$ to represent the $L^{2}(\Omega)$ inner product for scalar-, vectorand matrix-valued functions. The dual pairing between $S$ and the dual space $S^{\prime}$ is denoted by $\langle\cdot, \cdot\rangle$. The norms on $W^{m, p}(\Omega)$ and $H^{m}(\Omega)$ and their seminorms are simply denoted by $\|\cdot\|_{m, p}$ and $\|\cdot\|_{m}\left(=\|\cdot\|_{m, 2}\right)$ and by $|\cdot|_{m, p}$ and 
$|\cdot|_{m}\left(=|\cdot|_{m, 2}\right)$, respectively. The notations $\|\cdot\|_{m, p},|\cdot|_{m, p},\|\cdot\|_{m}$ and $|\cdot|_{m}$ are employed not only for scalar-valued functions but also for vector- and matrix-valued ones. We also denote the norm on $H^{-1}(\Omega)^{2}$ by $\|\cdot\|_{-1}$. For $t_{0}$ and $t_{1} \in \mathbb{R}$ we introduce the function space,

$$
Z^{m}\left(t_{0}, t_{1}\right):=\left\{\psi \in H^{j}\left(t_{0}, t_{1} ; H^{m-j}(\Omega)\right) ; j=0, \ldots, m,\|\psi\|_{Z^{m}\left(t_{0}, t_{1}\right)}<\infty\right\}
$$

with the norm

$$
\|\psi\|_{Z^{m}\left(t_{0}, t_{1}\right)}:=\left\{\sum_{j=0}^{m}\|\psi\|_{H^{j}\left(t_{0}, t_{1} ; H^{m-j}(\Omega)\right)}^{2}\right\}^{1 / 2},
$$

and set $Z^{m}:=Z^{m}(0, T)$. We often omit $[0, T], \Omega$, and the superscripts $d$ and $d \times d$ for the vector and the matrix if there is no confusion, e.g., we shall write $C\left(L^{\infty}\right)$ in place of $C\left([0, T] ; L^{\infty}(\Omega)^{d \times d}\right)$. For square matrices $\mathbf{A}$ and $\mathbf{B} \in \mathbb{R}^{d \times d}$ we use the notation $\mathbf{A}: \mathbf{B}:=\operatorname{tr}\left(\mathbf{A B}^{T}\right)=\sum_{i, j} A_{i j} B_{i j}$.

We consider the system of equations describing the unsteady motion of an incompressible viscoelastic fluid,

$$
\begin{aligned}
\frac{\mathrm{D} \mathbf{u}}{\mathrm{D} t}-\operatorname{div}(2 \nu \mathrm{D}(\mathbf{u}))+\nabla p & =\operatorname{div}[(\operatorname{tr} \mathbf{C}) \mathbf{C}]+\mathbf{f} & & \text { in } \Omega \times(0, T), \\
\operatorname{div} \mathbf{u} & =0 & & \text { in } \Omega \times(0, T), \\
\frac{\mathrm{D} \mathbf{C}}{\mathrm{D} t}-\varepsilon \Delta \mathbf{C}=(\nabla \mathbf{u}) \mathbf{C} & +\mathbf{C}(\nabla \mathbf{u})^{T}-(\operatorname{tr} \mathbf{C})^{2} \mathbf{C}+(\operatorname{tr} \mathbf{C}) \mathbf{I}+\mathbf{F} & & \text { in } \Omega \times(0, T), \\
\mathbf{u} & =\mathbf{0}, \quad \frac{\partial \mathbf{C}}{\partial \mathbf{n}}=\mathbf{0}, & & \text { on } \Gamma \times(0, T), \\
\mathbf{u} & =\mathbf{u}^{0}, \quad \mathbf{C}=\mathbf{C}^{0}, & & \text { in } \Omega, \text { at } t=0,
\end{aligned}
$$

where $(\mathbf{u}, p, \mathbf{C}): \Omega \times(0, T) \rightarrow \mathbb{R}^{d} \times \mathbb{R} \times \mathbb{R}_{s y m}^{d \times d}$ are the unknown velocity, pressure and conformation tensor, $\nu \in(0,1]$ is a fluid viscosity, $\varepsilon \in(0,1]$ is an elastic stress viscosity, $(\mathbf{f}, \mathbf{F}): \Omega \times(0, T) \rightarrow \mathbb{R}^{d} \times \mathbb{R}_{\text {sym }}^{d \times d}$ is a pair of given external forces, $\mathrm{D}(\mathbf{u}):=(1 / 2)\left[\nabla \mathbf{u}+(\nabla \mathbf{u})^{T}\right]$ is the symmetric part of the velocity gradient, $\mathbf{I}$ is the identity matrix, $\mathbf{n}: \Gamma \rightarrow \mathbb{R}^{d}$ is the outward unit normal, $\left(\mathbf{u}^{0}, \mathbf{C}^{0}\right): \Omega \rightarrow \mathbb{R}^{d} \times \mathbb{R}_{\text {sym }}^{d \times d}$ is a pair of given initial functions, and $\mathrm{D} / \mathrm{D} t$ is the material derivative defined by

$$
\frac{\mathrm{D}}{\mathrm{D} t}:=\frac{\partial}{\partial t}+\mathbf{w} \cdot \nabla
$$

where $\mathbf{w}: \Omega \times(0, T) \rightarrow \mathbb{R}^{d}$ is a given velocity.

Remark 1. The model (1) is the Oseen approximation to the fully nonlinear problem, where the material derivative terms,

$$
\frac{\partial \mathbf{u}}{\partial t}+(\mathbf{u} \cdot \nabla) \mathbf{u}, \quad \frac{\partial \mathbf{C}}{\partial t}+(\mathbf{u} \cdot \nabla) \mathbf{C}
$$

exist in place of $\frac{\mathrm{Du}}{\mathrm{D} t}$ and $\frac{\mathrm{DC}}{\mathrm{D} t}$ in equations (1a) and (1c). The existence of weak solutions and the uniqueness of regular solutions to the fully nonlinear model have been proved in Lukáčová-Medvid'ová et al. [30, Theorems 1 and 3]. The corresponding results are obtained under regularity condition on $\mathbf{w}$ to the model (1), which is simpler than the fully nonlinear model. Numerical analysis of the fully nonlinear problem is a future work.

We set an assumption for the given velocity $\mathbf{w}$.

Hypothesis 1. The function $\mathbf{w}$ satisfies $\mathbf{w} \in C\left([0, T] ; W_{0}^{1, \infty}(\Omega)^{d}\right)$.

Let $V:=H_{0}^{1}(\Omega)^{d}, Q:=L_{0}^{2}(\Omega)$ and $W:=H_{\text {sym }}^{1}(\Omega)$. We define the bilinear forms $a_{u}$ on $V \times V, b$ on $V \times Q, \mathcal{A}$ on $(V \times Q) \times(V \times Q)$ and $a_{c}$ on $W \times W$ by

$$
\begin{aligned}
a_{u}(\mathbf{u}, \mathbf{v}) & :=2(\mathrm{D}(\mathbf{u}), \mathrm{D}(\mathbf{v})), \quad b(\mathbf{u}, q):=-(\operatorname{div} \mathbf{u}, q), \quad \mathcal{A}((\mathbf{u}, p),(\mathbf{v}, q)):=\nu a_{u}(\mathbf{u}, \mathbf{v})+b(\mathbf{u}, q)+b(\mathbf{v}, p), \\
a_{c}(\mathbf{C}, \mathbf{D}) & :=(\nabla \mathbf{C}, \nabla \mathbf{D}),
\end{aligned}
$$

respectively. We present the weak formulation of the problem (1); find $(\mathbf{u}, p, \mathbf{C}):(0, T) \rightarrow V \times Q \times W$ such that for $t \in(0, T)$

$$
\left(\frac{\mathrm{D} \mathbf{u}}{\mathrm{D} t}(t), \mathbf{v}\right)+\mathcal{A}((\mathbf{u}, p)(t),(\mathbf{v}, q))=-(\operatorname{tr} \mathbf{C}(t) \mathbf{C}(t), \nabla \mathbf{v})+(\mathbf{f}(t), \mathbf{v})
$$




$$
\begin{array}{r}
\left(\frac{\mathrm{D} \mathbf{C}}{\mathrm{D} t}(t), \mathbf{D}\right)+\varepsilon a_{c}(\mathbf{C}(t), \mathbf{D})=2((\nabla \mathbf{u}(t)) \mathbf{C}(t), \mathbf{D})-\left((\operatorname{tr} \mathbf{C}(t))^{2} \mathbf{C}(t), \mathbf{D}\right)+(\operatorname{tr} \mathbf{C}(t) \mathbf{I}, \mathbf{D})+(\mathbf{F}(t), \mathbf{D}), \\
\forall(\mathbf{v}, q, \mathbf{D}) \in V \times Q \times W,
\end{array}
$$

with $(\mathbf{u}(0), \mathbf{C}(0))=\left(\mathbf{u}^{0}, \mathbf{C}^{0}\right)$.

\section{A linear stabilized Lagrange-Galerkin scheme}

The aim of this section is to present a linear stabilized Lagrange-Galerkin scheme for the model (1).

Let $\Delta t$ be a time increment, $N_{T}:=\lfloor T / \Delta t\rfloor$ the total number of time steps and $t^{n}:=n \Delta t$ for $n=0, \ldots, N_{T}$. Let $\mathbf{g}$ be a function defined in $\Omega \times(0, T)$ and $\mathbf{g}^{n}:=\mathbf{g}\left(\cdot, t^{n}\right)$. For the approximation of the material derivative we employ the first-order characteristics method,

$$
\frac{\mathrm{Dg}}{\mathrm{D} t}\left(x, t^{n}\right)=\frac{\mathbf{g}^{n}(x)-\left(\mathbf{g}^{n-1} \circ X_{1}^{n}\right)(x)}{\Delta t}+O(\Delta t)
$$

where $X_{1}^{n}: \Omega \rightarrow \mathbb{R}^{d}$ is a mapping defined by

$$
X_{1}^{n}(x):=x-\mathbf{w}^{n}(x) \Delta t
$$

and the symbol $\circ$ means the composition of functions,

$$
\left(\mathbf{g}^{n-1} \circ X_{1}^{n}\right)(x):=\mathbf{g}^{n-1}\left(X_{1}^{n}(x)\right) .
$$

For the details on deriving the approximation (3) of Dg/Dt, see, e.g., [38]. The point $X_{1}^{n}(x)$ is called the upwind point of $x$ with respect to $\mathbf{w}^{n}$. The next proposition, which is a direct consequence of [50] and [52], presents sufficient conditions to ensure that all upwind points defined by $X_{1}^{n}$ are in $\Omega$ and that its Jacobian $J^{n}:=\operatorname{det}\left(\partial X_{1}^{n} / \partial x\right)$ is around 1 .

Proposition 1. Suppose Hypothesis 1 holds. Then, we have the following for $n \in\left\{0, \ldots, N_{T}\right\}$.

(i) Under the condition

$$
\Delta t|\mathbf{w}|_{C\left(W^{1, \infty}\right)}<1
$$

$X_{1}^{n}: \Omega \rightarrow \Omega$ is bijective.

(ii) Furthermore, under the condition

$$
\Delta t|\mathbf{w}|_{C\left(W^{1, \infty}\right)} \leq 1 / 4
$$

the estimate $1 / 2 \leq J^{n} \leq 3 / 2$ holds.

For the sake of simplicity we suppose that $\Omega$ is a polygonal domain. Let $\mathcal{T}_{h}=\{K\}$ be a triangulation of $\bar{\Omega}(=$ $\left.\bigcup_{K \in \mathcal{T}_{h}} K\right), h_{K}$ the diameter of $K \in \mathcal{T}_{h}$ and $h:=\max _{K \in \mathcal{T}_{h}} h_{K}$ the maximum element size. We consider a regular family of subdivisions $\left\{\mathcal{T}_{h}\right\}_{h \downarrow 0}$ satisfying the inverse assumption [14], i.e., there exists a positive constant $\alpha_{0}$ independent of $h$ such that

$$
\frac{h}{h_{K}} \leq \alpha_{0}, \quad \forall K \in \mathcal{T}_{h}, \forall h .
$$

We define the discrete function spaces $X_{h}, M_{h}, W_{h}, V_{h}$ and $Q_{h}$ by

$$
\begin{aligned}
X_{h} & :=\left\{\mathbf{v}_{h} \in C(\bar{\Omega})^{d} ; \mathbf{v}_{h \mid K} \in P_{1}(K)^{d}, \forall K \in \mathcal{T}_{h}\right\}, \\
W_{h}:=\left\{\mathbf{D}_{h} \in C_{\text {sym }}(\bar{\Omega}) ; \mathbf{D}_{h \mid K} \in P_{1}(K)^{d \times d}, \forall K \in \mathcal{T}_{h}\right\}, & V_{h}:=\left\{q_{h} \in C(\bar{\Omega}) ; q_{h \mid K} \in P_{1}(K), \forall K \in \mathcal{T}_{h}\right\},
\end{aligned}
$$

respectively, where $P_{1}(K)$ is the polynomial space of linear functions on $K \in \mathcal{T}_{h}$.

Let $\delta_{0}$ be a small positive constant fixed arbitrarily and $(\cdot, \cdot)_{K}$ the $L^{2}(K)^{d}$ inner product. We define the bilinear forms $\mathcal{A}_{h}$ on $\left(V \times H^{1}(\Omega)\right) \times\left(V \times H^{1}(\Omega)\right)$ and $\mathcal{S}_{h}$ on $H^{1}(\Omega) \times H^{1}(\Omega)$ by

$$
\mathcal{A}_{h}((\mathbf{u}, p),(\mathbf{v}, q)):=\nu a_{u}(\mathbf{u}, \mathbf{v})+b(\mathbf{u}, q)+b(\mathbf{v}, p)-\mathcal{S}_{h}(p, q), \quad \mathcal{S}_{h}(p, q):=\delta_{0} \sum_{K \in \mathcal{T}_{h}} h_{K}^{2}(\nabla p, \nabla q)_{K} .
$$


Let $\left(\mathbf{f}_{h}, \mathbf{F}_{h}\right):=\left(\left\{\mathbf{f}_{h}^{n}\right\}_{n=1}^{N_{T}},\left\{\mathbf{F}_{h}^{n}\right\}_{n=1}^{N_{T}}\right) \subset L^{2}(\Omega)^{d} \times L^{2}(\Omega)^{d \times d}$ and $\left(\mathbf{u}_{h}^{0}, \mathbf{C}_{h}^{0}\right) \in V_{h} \times W_{h}$ be given. A linear stabilized Lagrange-Galerkin scheme for (1) is to find $\left(\mathbf{u}_{h}, p_{h}, \mathbf{C}_{h}\right):=\left\{\left(\mathbf{u}_{h}^{n}, p_{h}^{n}, \mathbf{C}_{h}^{n}\right)\right\}_{n=1}^{N_{T}} \subset V_{h} \times Q_{h} \times W_{h}$ such that, for $n=1, \ldots, N_{T}$,

$$
\begin{array}{r}
\left(\frac{\mathbf{u}_{h}^{n}-\mathbf{u}_{h}^{n-1} \circ X_{1}^{n}}{\Delta t}, \mathbf{v}_{h}\right)+\mathcal{A}_{h}\left(\left(\mathbf{u}_{h}^{n}, p_{h}^{n}\right),\left(\mathbf{v}_{h}, q_{h}\right)\right)=-\left(\left(\operatorname{tr} \mathbf{C}_{h}^{n}\right) \mathbf{C}_{h}^{n-1}, \nabla \mathbf{v}_{h}\right)+\left(\mathbf{f}_{h}^{n}, \mathbf{v}_{h}\right), \\
\left(\frac{\mathbf{C}_{h}^{n}-\mathbf{C}_{h}^{n-1} \circ X_{1}^{n}}{\Delta t}, \mathbf{D}_{h}\right)+\varepsilon a_{c}\left(\mathbf{C}_{h}^{n}, \mathbf{D}_{h}\right)=2\left(\left(\nabla \mathbf{u}_{h}^{n}\right) \mathbf{C}_{h}^{n-1}, \mathbf{D}_{h}\right)-\left(\left(\operatorname{tr} \mathbf{C}_{h}^{n-1}\right)^{2} \mathbf{C}_{h}^{n}, \mathbf{D}_{h}\right) \\
+\left(\left(\operatorname{tr} \mathbf{C}_{h}^{n-1}\right) \mathbf{I}, \mathbf{D}_{h}\right)+\left(\mathbf{F}_{h}^{n}, \mathbf{D}_{h}\right), \\
\forall\left(\mathbf{v}_{h}, q_{h}, \mathbf{D}_{h}\right) \in V_{h} \times Q_{h} \times W_{h} .
\end{array}
$$

\section{The main result}

In this section we state the main result on error estimates with the optimal convergence order of scheme (6), which is proved in the next section.

We use $c, c_{w}, c_{s}, c_{w, s}, c_{\nu}, c_{\varepsilon}$ and $c_{\nu, \varepsilon}$ to represent generic positive constants independent of the discretization parameters $h$ and $\Delta t$, the subscripts imply the dependency of the constants, and the subscripts " $w$ " and " $s$ " in $c_{w}, c_{s}$ and $c_{w, s}$ mean the given velocity $\mathbf{w}$ and the solution $(\mathbf{u}, p, \mathbf{C})$ of $(2)$, respectively. For instance, the constant $c_{w, s}$ is dependent on $\mathbf{w}$ and $(\mathbf{u}, p, \mathbf{C})$ and independent of $\nu$ and $\varepsilon$, and the constant $c$ has no dependency on $\mathbf{w},(\mathbf{u}, p, \mathbf{C}), \nu$ nor $\varepsilon$. The symbol "/ (prime)" is sometimes used in order to distinguish two constants, e.g., $c_{s}$ and $c_{s}^{\prime}$, from each other.

We use the following notation for the norms and seminorms, $\|\cdot\|_{V}=\|\cdot\|_{V_{h}}:=\|\cdot\|_{1},\|\cdot\|_{Q}=\|\cdot\|_{Q_{h}}:=\|\cdot\|_{0}$,

$$
\begin{aligned}
& \|(\mathbf{u}, \mathbf{C})\|_{Z^{2}\left(t_{0}, t_{1}\right)}:=\left\{\|\mathbf{u}\|_{Z^{2}\left(t_{0}, t_{1}\right)}^{2}+\|\mathbf{C}\|_{Z^{2}\left(t_{0}, t_{1}\right)}^{2}\right\}^{1 / 2}, \\
& \|\mathbf{u}\|_{\ell^{\infty}(X)}:=\max _{n=0, \ldots, N_{T}}\left\|\mathbf{u}^{n}\right\|_{X}, \quad\|\mathbf{u}\|_{\ell_{m}^{2}(X)}:=\left\{\Delta t \sum_{n=1}^{m}\left\|\mathbf{u}^{n}\right\|_{X}^{2}\right\}^{1 / 2}, \quad\|\mathbf{u}\|_{\ell^{2}(X)}:=\|\mathbf{u}\|_{\ell_{N_{T}}^{2}(X)}, \\
& |p|_{h}:=\left\{\sum_{K \in \mathcal{T}_{h}} h_{K}^{2}(\nabla p, \nabla p)_{K}\right\}^{1 / 2}, \quad|p|_{\ell_{m}^{2}\left(|\cdot|_{h}\right)}:=\left\{\Delta t \sum_{n=1}^{m}\left|p^{n}\right|_{h}^{2}\right\}^{1 / 2}, \quad|p|_{\ell^{2}\left(|\cdot|_{h}\right)}:=|p|_{\ell_{N_{T}}^{2}\left(|\cdot|_{h}\right)},
\end{aligned}
$$

for $m \in\left\{1, \cdots, N_{T}\right\}$ and $X=L^{\infty}(\Omega), L^{2}(\Omega)$ and $H^{1}(\Omega)$. $\bar{D}_{\Delta t}$ is the backward difference operator defined by $\bar{D}_{\Delta t} \mathbf{u}^{n}:=\left(\mathbf{u}^{n}-\mathbf{u}^{n-1}\right) / \Delta t$.

The existence and uniqueness of the solution of scheme (6) are ensured by the following proposition, which is also proved in the next section.

Proposition 2 (existence and uniqueness). Suppose Hypothesis 1 holds. Then, for any $h$ and $\Delta t$ satisfying (4) there exists a unique solution $\left(\mathbf{u}_{h}, p_{h}, \mathbf{C}_{h}\right) \subset V_{h} \times Q_{h} \times W_{h}$ of scheme (6).

We state the main results after preparing a projection and a hypothesis.

Definition 1 (Stokes-Poisson projection). For $(\mathbf{u}, p, \mathbf{C}) \in V \times Q \times W$ we define the Stokes-Poisson projection $\left(\hat{\mathbf{u}}_{h}, \hat{p}_{h}, \hat{\mathbf{C}}_{h}\right) \in V_{h} \times Q_{h} \times W_{h}$ of $(\mathbf{u}, p, \mathbf{C})$ by

$$
\begin{array}{r}
\mathcal{A}_{h}\left(\left(\hat{\mathbf{u}}_{h}, \hat{p}_{h}\right),\left(\mathbf{v}_{h}, q_{h}\right)\right)+a_{c}\left(\hat{\mathbf{C}}_{h}, \mathbf{D}_{h}\right)+\left(\hat{\mathbf{C}}_{h}, \mathbf{D}_{h}\right)=\mathcal{A}\left((\mathbf{u}, p),\left(\mathbf{v}_{h}, q_{h}\right)\right)+a_{c}\left(\mathbf{C}, \mathbf{D}_{h}\right)+\left(\mathbf{C}, \mathbf{D}_{h}\right), \\
\forall\left(\mathbf{v}_{h}, q_{h}, \mathbf{D}_{h}\right) \in V_{h} \times Q_{h} \times W_{h} .
\end{array}
$$

The Stokes-Poisson projection derives an operator $\Pi_{h}^{\mathrm{SP}}: V \times Q \times W \rightarrow V_{h} \times Q_{h} \times W_{h}$ defined by $\Pi_{h}^{\mathrm{SP}}(\mathbf{u}, p, \mathbf{C}):=$ $\left(\hat{\mathbf{u}}_{h}, \hat{p}_{h}, \hat{\mathbf{C}}_{h}\right)$. We denote the $i$-th component of $\Pi_{h}^{\mathrm{SP}}(\mathbf{u}, p, \mathbf{C})$ by $\left[\Pi_{h}^{\mathrm{SP}}(\mathbf{u}, p, \mathbf{C})\right]_{i}$ for $i=1,2,3$ and the pair of the first and third components $\left(\hat{\mathbf{u}}_{h}, \hat{\mathbf{C}}_{h}\right)=\left(\left[\Pi_{h}^{\mathrm{SP}}(\mathbf{u}, p, \mathbf{C})\right]_{1},\left[\Pi_{h}^{\mathrm{SP}}(\mathbf{u}, p, \mathbf{C})\right]_{3}\right)$ by $\left[\Pi_{h}^{\mathrm{SP}}(\mathbf{u}, p, \mathbf{C})\right]_{1,3}$ simply.

Remark 2. The identity (7) can be decoupled into the Stokes projection and the Poisson projection. For the simplicity of the notation we use (7) in the sequel. Since the Neumann boundary condition (1d) is imposed on $\mathbf{C}$, we use the Poisson projection corresponding to the operator $-\Delta+I$ for the unique solvability. 
Hypothesis 2. The solution $(\mathbf{u}, p, \mathbf{C})$ of (2) satisfies $\mathbf{u} \in Z^{2}(0, T)^{d} \cap H^{1}\left(0, T ; V \cap H^{2}(\Omega)^{d}\right) \cap C\left([0, T] ; W^{1, \infty}(\Omega)^{d}\right)$, $p \in H^{1}\left(0, T ; Q \cap H^{1}(\Omega)\right)$ and $\mathbf{C} \in Z^{2}(0, T)^{d \times d} \cap H^{1}\left(0, T ; W \cap H^{2}(\Omega)^{d \times d}\right)$.

Remark 3. Let us note that we assume a higher regularity of the exact solution than that of the weak solution. Such regularity is usually assumed in discussing the convergence rate of numerical solutions of partial differential equations. We remark that our recent theoretical result [30] shows that both velocity and conformation tensor belong to $L^{\infty}\left(H^{2}\right)$ for the fully nonlinear Peterlin model with $\varepsilon>0$. The result holds also for the Oseen-type Peterlin model with $\varepsilon>0$.

We now impose the conditions

$$
\left(\mathbf{u}_{h}^{0}, \mathbf{C}_{h}^{0}\right)=\left[\Pi_{h}^{\mathrm{SP}}\left(\mathbf{u}^{0}, 0, \mathbf{C}^{0}\right)\right]_{1,3}, \quad\left(\mathbf{f}_{h}, \mathbf{F}_{h}\right)=(\mathbf{f}, \mathbf{F}) .
$$

Remark 4. For the choice of $\left(\mathbf{u}_{h}^{0}, \mathbf{C}_{h}^{0}\right)$ we employ the Stokes-Poisson projection of $\left(\mathbf{u}^{0}, 0, \mathbf{C}^{0}\right)$ by (7) in (8), since the initial condition for the pressure is not given in (1). This choice does not lose any convergence order in our results below.

Theorem 1 (error estimates I). Suppose Hypotheses 1 and 2 hold. Then, there exist positive constants $h_{0}, c_{0}$ and $c_{\dagger}$ such that, for any pair $(h, \Delta t)$ satisfying

$$
h \in\left(0, h_{0}\right], \quad \Delta t \leq \begin{cases}c_{0}(1+|\log h|)^{-1 / 2} & (d=2), \\ c_{0} h^{1 / 2} & (d=3),\end{cases}
$$

the solution $\left(\mathbf{u}_{h}, p_{h}, \mathbf{C}_{h}\right)$ of scheme (6) with (8) is estimated as follows.

$$
\begin{gathered}
\left\|\mathbf{C}_{h}\right\|_{\ell^{\infty}\left(L^{\infty}\right)} \leq\|\mathbf{C}\|_{C\left(L^{\infty}\right)}+1, \\
\left\|\mathbf{u}_{h}-\mathbf{u}\right\|_{\ell^{\infty}\left(L^{2}\right)},\left\|\mathbf{u}_{h}-\mathbf{u}\right\|_{\ell^{2}\left(H^{1}\right)},\left|p_{h}-p\right|_{\ell^{2}\left(|\cdot|_{h}\right)},\left\|\mathbf{C}_{h}-\mathbf{C}\right\|_{\ell^{\infty}\left(H^{1}\right)},\left\|\bar{D}_{\Delta t} \mathbf{C}_{h}-\frac{\partial \mathbf{C}}{\partial t}\right\|_{\ell^{2}\left(L^{2}\right)} \leq c_{\dagger}(\Delta t+h) .
\end{gathered}
$$

Theorem 2 (error estimates II). Suppose Hypotheses 1 and 2 hold. Let $h_{0}$ and $c_{0}$ be the constants stated in Theorem 1. Then, there exists a positive constant $c_{\ddagger}$ such that, for any pair $(h, \Delta t)$ with $(9)$ the solution $\left(\mathbf{u}_{h}, p_{h}, \mathbf{C}_{h}\right)$ of scheme (6) with (8) satisfies the estimates,

$$
\left\|\bar{D}_{\Delta t} \mathbf{u}_{h}-\frac{\partial \mathbf{u}}{\partial t}\right\|_{\ell^{2}\left(L^{2}\right)}, \quad\left\|p_{h}-p\right\|_{\ell^{2}\left(L^{2}\right)} \leq c_{\ddagger}(\Delta t+h) .
$$

Remark 5. (i) The condition (9) is mild in comparison with, e.g., the CFL condition of the form $\|\mathbf{w}\|_{C\left(L^{\infty}\right)} \Delta t \leq$ ch. We can take $\Delta t=c^{\alpha}$ for any $\alpha>0(d=2)$ or $\alpha \geq 1 / 2(d=3)$.

(ii) The condition (9) is needed to deal with the nonlinearity of the model or, more precisely, to get the boundedness of $\left\|\mathbf{C}_{h}^{n}\right\|_{0, \infty}$ by using the inverse inequality (14), cf. the estimate (35) with (29a). In fact, the stabilized LagrangeGalerkin scheme for the Oseen equations is stable under only (5), cf. [38].

\section{Proofs}

In what follows we prove Proposition 2 and Theorems 1 and 2.

\subsection{Preliminaries}

Let us list lemmas employed directly in the proofs below. In the lemmas, $\alpha_{i}, i=1, \ldots, 4$, are numerical constants independent of $h, \Delta t, \nu$ and $\varepsilon$.

Lemma 1 ( [33] ). Let $\Omega$ be a bounded domain with a Lipschitz-continuous boundary. Then, the following inequalities hold.

$$
\|\mathrm{D}(\mathbf{v})\|_{0} \leq\|\mathbf{v}\|_{1} \leq \alpha_{1}\|\mathrm{D}(\mathbf{v})\|_{0}, \quad \forall \mathbf{v} \in H_{0}^{1}(\Omega)^{d} .
$$


Let $\Pi_{h}: C(\bar{\Omega}) \rightarrow M_{h}$ be the Lagrange interpolation operator. The operators defined on $C(\bar{\Omega})^{d}$ and $C(\bar{\Omega})^{d \times d}$ are also denoted by the same symbol $\Pi_{h}$. We introduce the function

$$
D(h):= \begin{cases}(1+|\log h|)^{1 / 2} & (d=2) \\ h^{-1 / 2} & (d=3)\end{cases}
$$

which is used in the sequel.

Lemma 2 ( $[11,14]$ ). The following inequalities hold.

$$
\begin{aligned}
\left\|\Pi_{h} \mathbf{D}\right\|_{0, \infty} & \leq\|\mathbf{D}\|_{0, \infty}, & & \mathbf{D} \in C(\bar{\Omega})^{d \times d}, \\
\left\|\Pi_{h} \mathbf{D}-\mathbf{D}\right\|_{1} & \leq \alpha_{20} h\|\mathbf{D}\|_{2}, & & \mathbf{D} \in H^{2}(\Omega)^{d \times d} \\
\left\|\mathbf{D}_{h}\right\|_{0, \infty} & \leq \alpha_{21} D(h)\left\|\mathbf{D}_{h}\right\|_{1}, & & \forall \mathbf{D}_{h} \in W_{h} .
\end{aligned}
$$

The next lemma is obtained by combining the error estimates for the Stokes and the Poisson problems, see, e.g., $[12,14,23]$ for the proof.

Lemma 3. (i) The following inequality holds.

$$
\inf _{\left(u_{h}, p_{h}\right) \in V_{h} \times Q_{h}} \sup _{\left(v_{h}, q_{h}\right) \in V_{h} \times Q_{h}} \frac{\mathcal{A}_{h}\left(\left(u_{h}, p_{h}\right),\left(v_{h}, q_{h}\right)\right)}{\left\|\left(u_{h}, p_{h}\right)\right\|_{V \times Q}\left\|\left(v_{h}, q_{h}\right)\right\|_{V \times Q}} \geq \nu \alpha_{30} .
$$

(ii) Assume $(\mathbf{u}, p, \mathbf{C}) \in\left(V \cap H^{2}(\Omega)^{d}\right) \times\left(Q \cap H^{1}(\Omega)\right) \times\left(W \cap H^{2}(\Omega)^{d \times d}\right)$. Let $\left(\hat{\mathbf{u}}_{h}, \hat{p}_{h}, \hat{\mathbf{C}}_{h}\right) \in V_{h} \times Q_{h} \times W_{h}$ be the Stokes-Poisson projection of $(\mathbf{u}, p, \mathbf{C})$ defined by $(7)$. Then, the following inequalities hold.

$$
\left\|\hat{\mathbf{u}}_{h}-\mathbf{u}\right\|_{1},\left\|\hat{p}_{h}-p\right\|_{0},\left|\hat{p}_{h}-p\right|_{h} \leq \frac{\alpha_{31}}{\nu} h\|(\mathbf{u}, p)\|_{H^{2} \times H^{1}}, \quad \quad\left\|\hat{\mathbf{C}}_{h}-\mathbf{C}\right\|_{1} \leq \alpha_{32} h\|\mathbf{C}\|_{2} .
$$

Remark 6. Let us note that the first part of error estimates in (ii) is based on the generalized inf-sup condition in (i) that is satisfied by the bilinear form $\mathcal{A}_{h}$ defined above and the pair of the discrete function spaces $V_{h}$ and $Q_{h}$, where the $\nu$-dependency is obtained by a simple modification of the analysis in, e.g., [23] after taking into account the diffusion constant.

Remark 7. As pointed out in [8], there are basically three possible approaches to obtain stable and convergent numerical methods for viscoelastic fluid flow problems. Firstly, the usual Galerkin methods using finite element spaces satisfying the inf-sup condition, e.g., [3, 22,34]. Secondly, the equal-order approximations for the velocity, pressure and stress with stabilization terms added to the usual weak formulation, see for instance [19]. And finally, the elastic viscous split stress (EVSS) method, e.g., [18,24,45], in which the stress is split into two parts, the elastic and the viscous part. Scheme (6) is classified into the second approach. Theorems 1 and 2 imply that our method for the Peterlin viscoelastic model is indeed stable and convergent.

Lemma 4 ( $[38,50])$. Under Hypothesis 1 and the condition (5) the following inequalities hold for any $n \in$ $\left\{0, \ldots, N_{T}\right\}$.

$$
\begin{aligned}
\left\|\mathbf{g} \circ X_{1}^{n}\right\|_{0} \leq\left(1+\alpha_{40}\left|\mathbf{w}^{n}\right|_{1, \infty} \Delta t\right)\|\mathbf{g}\|_{0}, & & \forall \mathbf{g} \in L^{2}(\Omega)^{s} \\
\left\|\mathbf{g}-\mathbf{g} \circ X_{1}^{n}\right\|_{0} \leq \alpha_{41}\left\|\mathbf{w}^{n}\right\|_{0, \infty} \Delta t|\mathbf{g}|_{1}, & & \forall \mathbf{g} \in H^{1}(\Omega)^{s}
\end{aligned}
$$

where $s=d$ or $d \times d$.

Proof. We prove only the former estimate, and see the proof of [38, Lemma 6] for the latter. Let $n \in\left\{0, \ldots, N_{T}\right\}$ be fixed arbitrarily. By changing the variable from $x$ to $y:=X_{1}^{n}(x)$, we have

$$
\left\|\mathbf{g} \circ X_{1}^{n}\right\|_{0}^{2}=\int_{\Omega} \mathbf{g}\left(X_{1}^{n}(x)\right)^{2} d x=\int_{\Omega} \mathbf{g}(y)^{2} \frac{1}{J^{n}} d y \leq\left(1+\alpha_{40}\left|\mathbf{w}^{n}\right|_{1, \infty} \Delta t\right)^{2}\|\mathbf{g}\|_{0}^{2},
$$

where $J^{n}$ is the Jacobian $\operatorname{det}(\partial y / \partial x)$. Here we have used the estimate,

$$
\frac{1}{J^{n}} \leq \frac{1}{1-\left|1-J^{n}\right|} \leq 1+2\left|1-J^{n}\right| \leq 1+2 \alpha_{40}\left|\mathbf{w}^{n}\right|_{1, \infty} \Delta t \leq\left(1+\alpha_{40}\left|\mathbf{w}^{n}\right|_{1, \infty} \Delta t\right)^{2}
$$

which is derived from Proposition 1 -(ii) and $1 /(1-s) \leq 1+2 s(s \in[0,1 / 2])$. 
We use the following simplified version of the discrete Gronwall inequality [25, Lemma 5.1].

Lemma 5. Let $\alpha$ and $\beta$ be non-negative numbers, $\Delta t$ a positive number, and $\left\{x^{n}\right\}_{n \geq 0}$ and $\left\{y^{n}\right\}_{n \geq 1}$ non-negative sequences. Suppose the inequality

$$
x^{m}+\Delta t \sum_{n=1}^{m} y^{n} \leq \alpha \Delta t \sum_{n=0}^{m-1} x^{n}+\beta, \quad \forall m \geq 0,
$$

holds. Then, it holds that

$$
x^{m}+\Delta t \sum_{n=1}^{m} y^{n} \leq(1+\alpha \Delta t)^{m} \beta, \quad \forall m \geq 0
$$

\subsection{Proof of Proposition 2}

For each time step $n$ scheme (6) can be rewritten as

$$
\begin{array}{rlrl}
\left(\frac{\mathbf{u}_{h}^{n}}{\Delta t}, \mathbf{v}_{h}\right)+\nu a_{u}\left(\mathbf{u}_{h}^{n}, \mathbf{v}_{h}\right)+b\left(\mathbf{v}_{h}, p_{h}^{n}\right)+\left(\left(\operatorname{tr} \mathbf{C}_{h}^{n}\right) \mathbf{C}_{h}^{n-1}, \nabla \mathbf{v}_{h}\right) & =\left(\mathbf{g}_{h}^{n}, \mathbf{v}_{h}\right), & & \forall \mathbf{v}_{h} \in V_{h}, \\
b\left(\mathbf{u}_{h}^{n}, q_{h}\right)-\mathcal{S}_{h}\left(p_{h}^{n}, q_{h}\right)=0, & & \forall q_{h} \in Q_{h}, \\
\left(\frac{\mathbf{C}_{h}^{n}}{\Delta t}, \mathbf{D}_{h}\right)+\varepsilon a_{c}\left(\mathbf{C}_{h}^{n}, \mathbf{D}_{h}\right)-2\left(\left(\nabla \mathbf{u}_{h}^{n}\right) \mathbf{C}_{h}^{n-1}, \mathbf{D}_{h}\right)+\left(\left(\operatorname{tr} \mathbf{C}_{h}^{n-1}\right)^{2} \mathbf{C}_{h}^{n}, \mathbf{D}_{h}\right)=\left(\mathbf{G}_{h}^{n}, \mathbf{D}_{h}\right), & & \forall \mathbf{D}_{h} \in W_{h},
\end{array}
$$

where $\mathbf{g}_{h}^{n}:=(1 / \Delta t)\left(\mathbf{u}_{h}^{n-1} \circ X_{1}^{n}\right)+\mathbf{f}_{h}^{n}$ and $\mathbf{G}_{h}^{n}:=(1 / \Delta t)\left(\mathbf{C}_{h}^{n-1} \circ X_{1}^{n}\right)+\left(\operatorname{tr} \mathbf{C}_{h}^{n-1}\right) \mathbf{I}+\mathbf{F}_{h}^{n}$. Selecting specific bases of $V_{h}, Q_{h}$ and $W_{h}$ and expanding $\mathbf{u}_{h}^{n}, p_{h}^{n}$ and $\mathbf{C}_{h}^{n}$ in terms of the associated basis functions, we can derive the system of linear equations from (15). The existence and uniqueness of the solution is equivalent to the invertibility of the coefficient matrix of the system, which is obtained by proving $\left(\mathbf{u}_{h}^{n}, p_{h}^{n}, \mathbf{C}_{h}^{n}\right)=(\mathbf{0}, 0, \mathbf{0})$ below when $\left(\mathbf{g}_{h}^{n}, \mathbf{G}_{h}^{n}\right)=(\mathbf{0}, \mathbf{0})$. Substituting $\left(\mathbf{u}_{h}^{n},-p_{h}^{n}, \frac{1}{2}\left(\operatorname{tr} \mathbf{C}_{h}^{n}\right) \mathbf{I}\right)$ into $\left(\mathbf{v}_{h}, q_{h}, \mathbf{D}_{h}\right)$ in (15) and adding (15b) to (15a), we have

$$
\begin{aligned}
& \frac{1}{\Delta t}\left\|\mathbf{u}_{h}^{n}\right\|_{0}^{2}+2 \nu\left\|\mathrm{D}\left(\mathbf{u}_{h}^{n}\right)\right\|_{0}^{2}+\delta_{0}\left|p_{h}^{n}\right|_{h}^{2}+\left(\left(\operatorname{tr} \mathbf{C}_{h}^{n}\right) \mathbf{C}_{h}^{n-1}, \nabla \mathbf{u}_{h}^{n}\right)=0, \\
& \frac{1}{2 \Delta t}\left\|\operatorname{tr} \mathbf{C}_{h}^{n}\right\|_{0}^{2}+\frac{\varepsilon}{2}\left\|\nabla \operatorname{tr} \mathbf{C}_{h}^{n}\right\|_{0}^{2}-\left(\operatorname{tr}\left[\left(\nabla \mathbf{u}_{h}^{n}\right) \mathbf{C}_{h}^{n-1}\right], \operatorname{tr} \mathbf{C}_{h}^{n}\right)+\frac{1}{2}\left\|\operatorname{tr} \mathbf{C}_{h}^{n-1} \operatorname{tr} \mathbf{C}_{h}^{n}\right\|_{0}^{2}=0 .
\end{aligned}
$$

By the identity

$$
\left(\left(\operatorname{tr} \mathbf{C}_{h}^{n}\right) \mathbf{C}_{h}^{n-1}, \nabla \mathbf{u}_{h}^{n}\right)-\left(\operatorname{tr}\left[\left(\nabla \mathbf{u}_{h}^{n}\right) \mathbf{C}_{h}^{n-1}\right], \operatorname{tr} \mathbf{C}_{h}^{n}\right)=0,
$$

the sum of $(16 \mathrm{a})$ and $(16 \mathrm{~b})$ yields

$$
\frac{1}{\Delta t}\left\|\mathbf{u}_{h}^{n}\right\|_{0}^{2}+2 \nu\left\|\mathrm{D}\left(\mathbf{u}_{h}^{n}\right)\right\|_{0}^{2}+\delta_{0}\left|p_{h}^{n}\right|_{h}^{2}+\frac{1}{2 \Delta t}\left\|\operatorname{tr} \mathbf{C}_{h}^{n}\right\|_{0}^{2}+\frac{\varepsilon}{2}\left\|\nabla \operatorname{tr} \mathbf{C}_{h}^{n}\right\|_{0}^{2}+\frac{1}{2}\left\|\operatorname{tr} \mathbf{C}_{h}^{n-1} \operatorname{tr} \mathbf{C}_{h}^{n}\right\|_{0}^{2}=0 .
$$

Hence, we have $\left(\mathbf{u}_{h}^{n}, p_{h}^{n}\right)=(\mathbf{0}, 0)$. Substituting $\mathbf{C}_{h}^{n}$ into $\mathbf{D}_{h}$ in $(15 \mathrm{c})$ and noting that $\mathbf{u}_{h}^{n}=\mathbf{0}$, we obtain

$$
\frac{1}{\Delta t}\left\|\mathbf{C}_{h}^{n}\right\|_{0}^{2}+\varepsilon\left\|\nabla \mathbf{C}_{h}^{n}\right\|_{0}^{2}+\left\|\left(\operatorname{tr} \mathbf{C}_{h}^{n-1}\right) \mathbf{C}_{h}^{n}\right\|_{0}^{2}=0
$$

which implies $\mathbf{C}_{h}^{n}=0$. Thus, we get $\left(\mathbf{u}_{h}^{n}, p_{h}^{n}, \mathbf{C}_{h}^{n}\right)=(\mathbf{0}, 0, \mathbf{0})$, which completes the proof.

\subsection{An estimate at each time step}

In this subsection we present a proposition which is employed in the proof of Theorem 1.

Let $\left(\hat{\mathbf{u}}_{h}, \hat{p}_{h}, \hat{\mathbf{C}}_{h}\right)(t):=\Pi_{h}^{\mathrm{SP}}(\mathbf{u}, p, \mathbf{C})(t) \in V_{h} \times Q_{h} \times W_{h}$ for $t \in[0, T]$ and let

$$
\mathbf{e}_{h}^{n}:=\mathbf{u}_{h}^{n}-\hat{\mathbf{u}}_{h}^{n}, \quad \epsilon_{h}^{n}:=p_{h}^{n}-\hat{p}_{h}^{n}, \quad \mathbf{E}_{h}^{n}:=\mathbf{C}_{h}^{n}-\hat{\mathbf{C}}_{h}^{n}, \quad \boldsymbol{\eta}(t):=\left(\mathbf{u}-\hat{\mathbf{u}}_{h}\right)(t), \quad \boldsymbol{\Xi}(t):=\left(\mathbf{C}-\hat{\mathbf{C}}_{h}\right)(t) .
$$

Then, from (6), (7) and (2), we have for $n \geq 1$

$$
\left(\frac{\mathbf{e}_{h}^{n}-\mathbf{e}_{h}^{n-1} \circ X_{1}^{n}}{\Delta t}, \mathbf{v}_{h}\right)+\mathcal{A}_{h}\left(\left(\mathbf{e}_{h}^{n}, \epsilon_{h}^{n}\right),\left(\mathbf{v}_{h}, q_{h}\right)\right)=\left\langle\mathbf{r}_{h}^{n}, \mathbf{v}_{h}\right\rangle, \quad \forall\left(\mathbf{v}_{h}, q_{h}\right) \in V_{h} \times Q_{h},
$$




$$
\left(\frac{\mathbf{E}_{h}^{n}-\mathbf{E}_{h}^{n-1} \circ X_{1}^{n}}{\Delta t}, \mathbf{v}_{h}\right)+\varepsilon a_{c}\left(\mathbf{E}_{h}^{n}, \mathbf{D}_{h}\right)=\left\langle\mathbf{R}_{h}^{n}, \mathbf{D}_{h}\right\rangle, \quad \forall \mathbf{D}_{h} \in W_{h},
$$

where

$$
\begin{aligned}
\mathbf{r}_{h}^{n} & :=\sum_{i=1}^{4} \mathbf{r}_{h i}^{n} \in V_{h}^{\prime}, \quad \mathbf{R}_{h}^{n}:=\sum_{i=1}^{11} \mathbf{R}_{h i}^{n} \in W_{h}^{\prime}, \\
\left\langle\mathbf{r}_{h 1}^{n}, \mathbf{v}_{h}\right\rangle & :=\left(\frac{\mathrm{D} \mathbf{u}^{n}}{\mathrm{D} t}-\frac{\mathbf{u}^{n}-\mathbf{u}^{n-1} \circ X_{1}^{n}}{\Delta t}, \mathbf{v}_{h}\right), \\
\left\langle\mathbf{r}_{h 2}^{n}, \mathbf{v}_{h}\right\rangle & :=\frac{1}{\Delta t}\left(\boldsymbol{\eta}^{n}-\boldsymbol{\eta}^{n-1} \circ X_{1}^{n}, \mathbf{v}_{h}\right), \\
\left\langle\mathbf{r}_{h 3}^{n}, \mathbf{v}_{h}\right\rangle & :=\left(\left(\operatorname{tr} \mathbf{C}^{n}\right)\left(\mathbf{C}^{n}-\mathbf{C}^{n-1}+\mathbf{\Xi}^{n-1}-\mathbf{E}_{h}^{n-1}\right), \nabla \mathbf{v}_{h}\right), \\
\left\langle\mathbf{r}_{h 4}^{n}, \mathbf{v}_{h}\right\rangle & :=\left(\left[\operatorname{tr}\left(\mathbf{\Xi}^{n}-\mathbf{E}_{h}^{n}\right)\right] \mathbf{C}_{h}^{n-1}, \nabla \mathbf{v}_{h}\right), \\
\left\langle\mathbf{R}_{h 1}^{n}, \mathbf{D}_{h}\right\rangle & :=\left(\frac{\mathrm{D} \mathbf{C}^{n}}{\mathrm{D} t}-\frac{\mathbf{C}^{n}-\mathbf{C}^{n-1} \circ X_{1}^{n}}{\Delta t}, \mathbf{D}_{h}\right), \\
\left\langle\mathbf{R}_{h 2}^{n}, \mathbf{D}_{h}\right\rangle & :=\frac{1}{\Delta t}\left(\boldsymbol{\Xi}^{n}-\boldsymbol{\Xi}^{n-1} \circ X_{1}^{n}, \mathbf{D}_{h}\right), \\
\left\langle\mathbf{R}_{h 3}^{n}, \mathbf{D}_{h}\right\rangle & :=-\varepsilon\left(\mathbf{\Xi}^{n}, \mathbf{D}_{h}\right), \\
\left\langle\mathbf{R}_{h 4}^{n}, \mathbf{D}_{h}\right\rangle & :=2\left(\left(\nabla \mathbf{e}_{h}^{n}\right) \mathbf{C}_{h}^{n-1}, \mathbf{D}_{h}\right), \\
\left\langle\mathbf{R}_{h 5}^{n}, \mathbf{D}_{h}\right\rangle & :=-2\left(\left(\nabla \boldsymbol{\eta}^{n}\right) \mathbf{C}_{h}^{n-1}, \mathbf{D}_{h}\right), \\
\left\langle\mathbf{R}_{h 6}^{n}, \mathbf{D}_{h}\right\rangle & :=-2\left(\left(\nabla \mathbf{u}^{n}\right)\left(\mathbf{C}^{n}-\mathbf{C}^{n-1}+\mathbf{\Xi}^{n-1}-\mathbf{E}_{h}^{n-1}\right), \mathbf{D}_{h}\right), \\
\left\langle\mathbf{R}_{h 7}^{n}, \mathbf{D}_{h}\right\rangle & :=\left(\left(\operatorname{tr} \mathbf{C}_{h}^{n-1}\right)^{2}\left(\mathbf{\Xi}^{n}-\mathbf{E}_{h}^{n}\right), \mathbf{D}_{h}\right), \\
\left\langle\mathbf{R}_{h 8}^{n}, \mathbf{D}_{h}\right\rangle & :=-\left(\left[\operatorname{tr}\left(\mathbf{C}_{h}^{n-1}+\hat{\mathbf{C}}_{h}^{n-1}\right)\right]\left(\operatorname{tr} \mathbf{E}_{h}^{n-1}\right) \mathbf{C}^{n}, \mathbf{D}_{h}\right), \\
\left\langle\mathbf{R}_{h 9}^{n}, \mathbf{D}_{h}\right\rangle & :=\left(\left[\operatorname{tr}\left(\mathbf{C}^{n-1}+\hat{\mathbf{C}}_{h}^{n-1}\right)\right]\left(\operatorname{tr} \mathbf{\Xi}^{n-1}\right) \mathbf{C}^{n}, \mathbf{D}_{h}\right), \\
\left\langle\mathbf{R}_{h 10}^{n}, \mathbf{D}_{h}\right\rangle & :=\left(\left[\operatorname{tr}\left(\mathbf{C}^{n}+\mathbf{C}^{n-1}\right)\right]\left[\operatorname{tr}\left(\mathbf{C}^{n}-\mathbf{C}^{n-1}\right)\right] \mathbf{C}^{n}, \mathbf{D}_{h}\right), \\
\left\langle\mathbf{R}_{h 11}^{n}, \mathbf{D}_{h}\right\rangle & :=-\left(\left[\operatorname{tr}\left(\mathbf{C}^{n}-\mathbf{C}^{n-1}+\boldsymbol{\Xi}^{n-1}-\mathbf{E}_{h}^{n-1}\right)\right] \mathbf{I}, \mathbf{D}_{h}\right) .
\end{aligned}
$$

We note that

$$
\left(\mathbf{e}_{h}^{0}, \mathbf{E}_{h}^{0}\right)=\left(\mathbf{u}_{h}^{0}, \mathbf{C}_{h}^{0}\right)-\left(\hat{\mathbf{u}}_{h}^{0}, \hat{\mathbf{C}}_{h}^{0}\right)=\left[\Pi_{h}^{\mathrm{SP}}\left(0,-p^{0}, 0\right)\right]_{1,3} .
$$

In the following we use the constants $\alpha_{i}$ defined in Lemma $i, i=1, \ldots, 4$, and the notation $\mathbb{H}^{2}:=H^{2}(\Omega)^{2} \times$ $H^{1}(\Omega) \times H^{2}(\Omega)^{2 \times 2}$.

Proposition 3. Suppose that Hypotheses 1 and 2 hold and assume (5). Let $M_{0} \geq 1$ be a positive constant independent of $h$ and $\Delta t$. Let $\left(\mathbf{u}_{h}, p_{h}, \mathbf{C}_{h}\right)$ be the solution of scheme (6) with (8). Suppose that for an $n \in$ $\left\{1, \ldots, N_{T}\right\}$

$$
\left\|\mathbf{C}_{h}^{n-1}\right\|_{0, \infty} \leq M_{0}
$$

Then, there exist positive constants $c_{1}$ and $c_{2}$, dependent on $M_{0}, \nu$ and $\varepsilon$ but independent of $h$ and $\Delta t$, such that

$$
\begin{aligned}
\bar{D}_{\Delta t} & \left(\frac{1}{2}\left\|\mathbf{e}_{h}^{n}\right\|_{0}^{2}+\frac{1}{2}\left\|\mathbf{E}_{h}^{n}\right\|_{0}^{2}+\frac{\nu \varepsilon}{64 \alpha_{1}^{2} d^{2} M_{0}^{2}}\left|\mathbf{E}_{h}^{n}\right|_{1}^{2}\right)+\frac{\nu}{2 \alpha_{1}^{2}}\left\|\mathbf{e}_{h}^{n}\right\|_{1}^{2}+\delta_{0}\left|\epsilon_{h}^{n}\right|_{h}^{2}+\frac{\nu}{64 \alpha_{1}^{2} d^{2} M_{0}^{2}}\left\|\bar{D}_{\Delta t} \mathbf{E}_{h}^{n}\right\|_{0}^{2} \\
\leq & c_{1}\left(\frac{1}{2}\left\|\mathbf{e}_{h}^{n-1}\right\|_{0}^{2}+\frac{1}{2}\left\|\mathbf{E}_{h}^{n-1}\right\|_{0}^{2}+\frac{\nu \varepsilon}{64 \alpha_{1}^{2} d^{2} M_{0}^{2}}\left|\mathbf{E}_{h}^{n-1}\right|_{1}^{2}+\frac{1}{2}\left\|\mathbf{E}_{h}^{n}\right\|_{0}^{2}\right) \\
& +c_{2}\left[\Delta t\|(\mathbf{u}, \mathbf{C})\|_{Z^{2}\left(t^{n-1}, t^{n}\right)}^{2}+h^{2}\left(\frac{1}{\Delta t}\|(\mathbf{u}, p, \mathbf{C})\|_{H^{1}\left(t^{n-1}, t^{n} ; \mathbb{H}^{2}\right)}^{2}+1\right)\right] .
\end{aligned}
$$

For the proof we use the next lemma, which is proved in Appendix A.1.

Lemma 6. Suppose Hypotheses 1 and 2 hold. Let $n \in\left\{1, \ldots, N_{T}\right\}$ be any fixed number. Then, under the condition (5) it holds that

$$
\left\|\mathbf{r}_{h 1}^{n}\right\|_{0} \leq c_{w} \sqrt{\Delta t}\|\mathbf{u}\|_{Z^{2}\left(t^{n-1}, t^{n}\right)}
$$




$$
\begin{aligned}
\left\|\mathbf{r}_{h 2}^{n}\right\|_{0} & \leq \frac{c_{w} h}{\nu \sqrt{\Delta t}}\|(\mathbf{u}, p)\|_{H^{1}\left(t^{n-1}, t^{n} ; H^{2} \times H^{1}\right)}, \\
\left\|\mathbf{r}_{h 3}^{n}\right\|_{-1} & \leq c_{s}\left(\left\|\mathbf{E}_{h}^{n-1}\right\|_{0}+\sqrt{\Delta t}\|\mathbf{C}\|_{H^{1}\left(t^{n-1}, t^{n} ; L^{2}\right)}+h\right), \\
\left\|\mathbf{r}_{h 4}^{n}\right\|_{-1} & \leq c_{s}\left\|\mathbf{C}_{h}^{n-1}\right\|_{0, \infty}\left(\left\|\mathbf{E}_{h}^{n}\right\|_{0}+h\right), \\
\left\|\mathbf{R}_{h 1}^{n}\right\|_{0} & \leq c_{w} \sqrt{\Delta t}\|\mathbf{C}\|_{Z^{2}\left(t^{n-1}, t^{n}\right)}, \\
\left\|\mathbf{R}_{h 2}^{n}\right\|_{0} & \leq \frac{c_{w} h}{\sqrt{\Delta t}}\|\mathbf{C}\|_{H^{1}\left(t^{n-1}, t^{n} ; H^{2}\right)}, \\
\left\|\mathbf{R}_{h 3}^{n}\right\|_{0} & \leq c_{s} h, \\
\left\|\mathbf{R}_{h 4}^{n}\right\|_{0} & \leq 2 d\left\|\mathbf{C}_{h}^{n-1}\right\|_{0, \infty}\left\|\mathbf{e}_{h}^{n}\right\|_{1}, \\
\left\|\mathbf{R}_{h 5}^{n}\right\|_{0} & \leq c_{s}\left\|\mathbf{C}_{h}^{n-1}\right\|_{0, \infty} h, \\
\left\|\mathbf{R}_{h 6}^{n}\right\|_{0} & \leq c_{s}\left(\left\|\mathbf{E}_{h}^{n-1}\right\|_{0}+\sqrt{\Delta t}\|\mathbf{C}\|_{H^{1}\left(t^{n-1}, t^{n} ; L^{2}\right)}+h\right), \\
\left\|\mathbf{R}_{h 7}^{n}\right\|_{0} & \leq c_{s}\left\|\mathbf{C}_{h}^{n-1}\right\|_{0, \infty}^{2}\left(\left\|\mathbf{E}_{h}^{n}\right\|_{0}+h\right), \\
\left\|\mathbf{R}_{h 8}^{n}\right\|_{0} & \leq c_{s}\left(\left\|\mathbf{C}_{h}^{n-1}\right\|_{0, \infty}+1\right)\left\|\mathbf{E}_{h}^{n-1}\right\|_{0}, \\
\left\|\mathbf{R}_{h 9}^{n}\right\|_{0} & \leq c_{s} h, \\
\left\|\mathbf{R}_{h 10}^{n}\right\|_{0} & \leq c_{s} \sqrt{\Delta t}\|\mathbf{C}\|_{H^{1}\left(t^{n-1}, t^{n} ; L^{2}\right)}, \\
\left\|\mathbf{R}_{h 11}^{n}\right\|_{0} & \leq c_{s}\left(\left\|\mathbf{E}_{h}^{n-1}\right\|_{0}+\sqrt{\Delta t}\|\mathbf{C}\|_{H^{1}\left(t^{n-1}, t^{n} ; L^{2}\right)}+h\right) .
\end{aligned}
$$

Proof of Proposition 3. Substituting $\left(\mathbf{e}_{h}^{n},-\epsilon_{h}^{n}\right)$ into $\left(\mathbf{v}_{h}, q_{h}\right)$ in (17a) and noting that

$$
\begin{aligned}
\left(\frac{\mathbf{e}_{h}^{n}-\mathbf{e}_{h}^{n-1} \circ X_{1}^{n}}{\Delta t}, \mathbf{e}_{h}^{n}\right) & \geq \frac{1}{2 \Delta t}\left(\left\|\mathbf{e}_{h}^{n}\right\|_{0}^{2}-\left\|\mathbf{e}_{h}^{n-1} \circ X_{1}^{n}\right\|_{0}^{2}\right) \geq \frac{1}{2 \Delta t}\left[\left\|\mathbf{e}_{h}^{n}\right\|_{0}^{2}-\left(1+\alpha_{40}\left|\mathbf{w}^{n}\right|_{1, \infty} \Delta t\right)^{2}\left\|\mathbf{e}_{h}^{n-1}\right\|_{0}^{2}\right] \\
& \geq \bar{D}_{\Delta t}\left(\frac{1}{2}\left\|\mathbf{e}_{h}^{n}\right\|_{0}^{2}\right)-c_{w}\left\|\mathbf{e}_{h}^{n-1}\right\|_{0}^{2}, \\
\mathcal{A}_{h}\left(\left(\mathbf{e}_{h}^{n}, \epsilon_{h}^{n}\right),\left(\mathbf{e}_{h}^{n},-\epsilon_{h}^{n}\right)\right) & \geq \frac{2 \nu}{\alpha_{1}^{2}}\left\|\mathbf{e}_{h}^{n}\right\|_{1}^{2}+\delta_{0}\left|p_{h}^{n}\right|_{h}^{2}, \\
\left\langle\mathbf{r}_{h}^{n}, \mathbf{e}_{h}^{n}\right\rangle & \leq\left\|\mathbf{r}_{h}^{n}\right\|_{-1}\left\|\mathbf{e}_{h}^{n}\right\|_{1} \leq \frac{\alpha_{1}^{2}}{4 \nu}\left\|\mathbf{r}_{h}^{n}\right\|_{-1}^{2}+\frac{\nu}{\alpha_{1}^{2}}\left\|\mathbf{e}_{h}^{n}\right\|_{1}^{2},
\end{aligned}
$$

we have

$$
\bar{D}_{\Delta t}\left(\frac{1}{2}\left\|\mathbf{e}_{h}^{n}\right\|_{0}^{2}\right)+\frac{\nu}{\alpha_{1}^{2}}\left\|\mathbf{e}_{h}^{n}\right\|_{1}^{2}+\delta_{0}\left|\epsilon_{h}^{n}\right|_{h}^{2} \leq \frac{\alpha_{1}^{2}}{4 \nu}\left\|\mathbf{r}_{h}^{n}\right\|_{-1}^{2}+c_{w}\left\|\mathbf{e}_{h}^{n-1}\right\|_{0}^{2} .
$$

Similarly, substituting $\mathbf{E}_{h}^{n}$ and $\bar{D}_{\Delta t} \mathbf{E}_{h}^{n}$ into $\mathbf{D}_{h}$ in (17b) and noting that

$$
\begin{aligned}
\left(\frac{\mathbf{E}_{h}^{n}-\mathbf{E}_{h}^{n-1} \circ X_{1}^{n}}{\Delta t}, \mathbf{E}_{h}^{n}\right) & \geq \bar{D}_{\Delta t}\left(\frac{1}{2}\left\|\mathbf{E}_{h}^{n}\right\|_{0}^{2}\right)-c_{w}\left\|\mathbf{E}_{h}^{n-1}\right\|_{0}^{2}, \\
\varepsilon a_{c}\left(\mathbf{E}_{h}^{n}, \mathbf{E}_{h}^{n}\right) & =\varepsilon\left|\mathbf{E}_{h}^{n}\right|_{1}^{2} \geq 0, \\
\left\langle\mathbf{R}_{h}^{n}, \mathbf{E}_{h}^{n}\right\rangle & \leq\left\|\mathbf{R}_{h}^{n}\right\|_{0}\left\|\mathbf{E}_{h}^{n}\right\|_{0} \leq \sum_{i \in\{1, \ldots, 11\} \backslash\{4\}}\left\|\mathbf{R}_{h i}^{n}\right\|_{0}\left\|\mathbf{E}_{h}^{n}\right\|_{0}+\left\|\mathbf{R}_{h 4}^{n}\right\|_{0}\left\|\mathbf{E}_{h}^{n}\right\|_{0} \\
& \leq \sum_{i \in\{1, \ldots, 11\} \backslash\{4\}}\left(\frac{5}{2}\left\|\mathbf{R}_{h i}^{n}\right\|_{0}^{2}+\frac{1}{10}\left\|\mathbf{E}_{h}^{n}\right\|_{0}^{2}\right)+2 d M_{0}\left\|\mathbf{e}_{h}^{n}\right\|_{1}\left\|\mathbf{E}_{h}^{n}\right\|_{0} \quad(\text { by }(22 \mathrm{~h}),(20)) \\
& \leq \frac{5}{2} \sum_{i \in\{1, \ldots, 11\} \backslash\{4\}}\left\|\mathbf{R}_{h i}^{n}\right\|_{0}^{2}+\left\|\mathbf{E}_{h}^{n}\right\|_{0}^{2}+\frac{\nu}{4 \alpha_{1}^{2}}\left\|\mathbf{e}_{h}^{n}\right\|_{1}^{2}+\frac{4 \alpha_{1}^{2} d^{2} M_{0}^{2}}{\nu}\left\|\mathbf{E}_{h}^{n}\right\|_{0}^{2} \\
& =\frac{5}{2} \sum_{i \in\{1, \ldots, 11\} \backslash\{4\}}\left\|\mathbf{R}_{h i}^{n}\right\|_{0}^{2}+\left(1+\frac{4 \alpha_{1}^{2} d^{2} M_{0}^{2}}{\nu}\right)\left\|\mathbf{E}_{h}^{n}\right\|_{0}^{2}+\frac{\nu}{4 \alpha_{1}^{2}}\left\|\mathbf{e}_{h}^{n}\right\|_{1}^{2}, \\
\left(\frac{\mathbf{E}_{h}^{n}-\mathbf{E}_{h}^{n-1} \circ X_{1}^{n}}{\Delta t}, \bar{D}_{\Delta t} \mathbf{E}_{h}^{n}\right) & =\left(\bar{D}_{\Delta t} \mathbf{E}_{h}^{n}+\frac{\mathbf{E}_{h}^{n-1}-\mathbf{E}_{h}^{n-1} \circ X_{1}^{n}}{\Delta t}, \bar{D}_{\Delta t} \mathbf{E}_{h}^{n}\right) \\
& \geq\left\|\bar{D}_{\Delta t} \mathbf{E}_{h}^{n}\right\|_{0}^{2}-\alpha_{41}\left\|\mathbf{w}^{n}\right\|_{0, \infty}\left|\mathbf{E}_{h}^{n-1}\right|_{1}\left\|\bar{D}_{\Delta t} \mathbf{E}_{h}^{n}\right\|_{0},
\end{aligned}
$$




$$
\begin{aligned}
& \geq\left\|\bar{D}_{\Delta t} \mathbf{E}_{h}^{n}\right\|_{0}^{2}-c_{w}\left|\mathbf{E}_{h}^{n-1}\right|_{1}^{2}-\frac{1}{4}\left\|\bar{D}_{\Delta t} \mathbf{E}_{h}^{n}\right\|_{0}^{2}, \\
& =\frac{3}{4}\left\|\bar{D}_{\Delta t} \mathbf{E}_{h}^{n}\right\|_{0}^{2}-c_{w}\left|\mathbf{E}_{h}^{n-1}\right|_{1}^{2}, \\
\varepsilon a_{c}\left(\mathbf{E}_{h}^{n}, \bar{D}_{\Delta t} \mathbf{E}_{h}^{n}\right) & \geq \bar{D}_{\Delta t}\left(\frac{\varepsilon}{2}\left|\mathbf{E}_{h}^{n}\right|_{1}^{2}\right), \\
\left\langle\mathbf{R}_{h}^{n}, \bar{D}_{\Delta t} \mathbf{E}_{h}^{n}\right\rangle & \leq\left\|\mathbf{R}_{h}^{n}\right\|_{0}\left\|\bar{D}_{\Delta t} \mathbf{E}_{h}^{n}\right\|_{0} \leq \sum_{i \in\{1, \ldots, 11\} \backslash\{4\}}\left\|\mathbf{R}_{h i}^{n}\right\|_{0}\left\|\bar{D}_{\Delta t} \mathbf{E}_{h}^{n}\right\|_{0}+\left\|\mathbf{R}_{h 4}^{n}\right\|_{0}\left\|\bar{D}_{\Delta t} \mathbf{E}_{h}^{n}\right\|_{0} \\
& \left.\leq \sum_{i \in\{1, \ldots, 11\} \backslash\{4\}}\left(20\left\|\mathbf{R}_{h i}^{n}\right\|_{0}^{2}+\frac{1}{80}\left\|\bar{D}_{\Delta t} \mathbf{E}_{h}^{n}\right\|_{0}^{2}\right)+2 d M_{0}\left\|\mathbf{e}_{h}^{n}\right\|_{1}\left\|\bar{D}_{\Delta t} \mathbf{E}_{h}^{n}\right\|_{0} \quad \text { by }(22 \mathrm{~h}),(20)\right) \\
& \leq 20 \sum_{i \in\{1, \ldots, 11\} \backslash\{4\}}\left\|\mathbf{R}_{h i}^{n}\right\|_{0}^{2}+\frac{1}{8}\left\|\bar{D}_{\Delta t} \mathbf{E}_{h}^{n}\right\|_{0}^{2}+8 d^{2} M_{0}^{2}\left\|\mathbf{e}_{h}^{n}\right\|_{1}^{2}+\frac{1}{8}\left\|\bar{D}_{\Delta t} \mathbf{E}_{h}^{n}\right\|_{0}^{2} \\
& =20 \sum_{i \in\{1, \ldots, 11\} \backslash\{4\}}\left\|\mathbf{R}_{h i}^{n}\right\|_{0}^{2}+\frac{1}{4}\left\|\bar{D}_{\Delta t} \mathbf{E}_{h}^{n}\right\|_{0}^{2}+8 d^{2} M_{0}^{2}\left\|\mathbf{e}_{h}^{n}\right\|_{1}^{2},
\end{aligned}
$$

we have the following two inequalities,

$$
\begin{aligned}
& \bar{D}_{\Delta t}\left(\frac{1}{2}\left\|\mathbf{E}_{h}^{n}\right\|_{0}^{2}\right) \leq \frac{5}{2} \sum_{i \in\{1, \ldots, 11\} \backslash\{4\}}\left\|\mathbf{R}_{h i}^{n}\right\|_{0}^{2}+\left(1+\frac{4 \alpha_{1}^{2} d^{2} M_{0}^{2}}{\nu}\right)\left\|\mathbf{E}_{h}^{n}\right\|_{0}^{2}+c_{w}\left\|\mathbf{E}_{h}^{n-1}\right\|_{0}^{2}+\frac{\nu}{4 \alpha_{1}^{2}}\left\|\mathbf{e}_{h}^{n}\right\|_{1}^{2}, \\
& \bar{D}_{\Delta t}\left(\frac{\varepsilon}{2}\left|\mathbf{E}_{h}^{n}\right|_{1}^{2}\right)+\frac{1}{2}\left\|\bar{D}_{\Delta t} \mathbf{E}_{h}^{n}\right\|_{0}^{2} \leq 20 \sum_{i \in\{1, \ldots, 11\} \backslash\{4\}}\left\|\mathbf{R}_{h i}^{n}\right\|_{0}^{2}+c_{w}\left|\mathbf{E}_{h}^{n-1}\right|_{1}^{2}+8 d^{2} M_{0}^{2}\left\|\mathbf{e}_{h}^{n}\right\|_{1}^{2} .
\end{aligned}
$$

Lemma 6, (18) and (20) imply that

$$
\begin{gathered}
\left\|\mathbf{r}_{h}^{n}\right\|_{-1}^{2} \leq c_{w, s}\left(M_{0}^{2}\left\|\mathbf{E}_{h}^{n}\right\|_{0}^{2}+\left\|\mathbf{E}_{h}^{n-1}\right\|_{0}^{2}\right) \\
+\frac{c_{w, s}^{\prime}}{\nu}\left[\Delta t\|(\mathbf{u}, \mathbf{C})\|_{Z^{2}\left(t^{n-1}, t^{n}\right)}^{2}+h^{2}\left(\frac{1}{\Delta t}\|(\mathbf{u}, p)\|_{H^{1}\left(t^{n-1}, t^{n} ; H^{2} \times H^{1}\right)}^{2}+M_{0}^{2}+1\right)\right], \\
\sum_{i \in\{1, \ldots, 11\} \backslash\{4\}}\left\|\mathbf{R}_{h i}^{n}\right\|_{0}^{2} \leq c_{w, s}\left[M_{0}^{4}\left\|\mathbf{E}_{h}^{n}\right\|_{0}^{2}+\left(M_{0}^{2}+1\right)\left\|\mathbf{E}_{h}^{n-1}\right\|_{0}^{2}\right] \\
+c_{w, s}^{\prime}\left[\Delta t\|\mathbf{C}\|_{Z^{2}\left(t^{n-1}, t^{n}\right)}^{2}+h^{2}\left(\frac{1}{\Delta t}\|\mathbf{C}\|_{H^{1}\left(t^{n-1}, t^{n} ; H^{2}\right)}^{2}+M_{0}^{4}+M_{0}^{2}+1\right)\right] .
\end{gathered}
$$

Multiplying (24b) by $\nu /\left(32 \alpha_{1}^{2} d^{2} M_{0}^{2}\right)$, adding it and (24a) to (23) and using (25), we get

$$
\begin{aligned}
& \bar{D}_{\Delta t}\left(\frac{1}{2}\left\|\mathbf{e}_{h}^{n}\right\|_{0}^{2}+\frac{1}{2}\left\|\mathbf{E}_{h}^{n}\right\|_{0}^{2}+\frac{\nu \varepsilon}{64 \alpha_{1}^{2} d^{2} M_{0}^{2}}\left|\mathbf{E}_{h}^{n}\right|_{1}^{2}\right)+\frac{\nu}{2 \alpha_{1}^{2}}\left\|\mathbf{e}_{h}^{n}\right\|_{1}^{2}+\delta_{0}\left|\epsilon_{h}^{n}\right|_{h}^{2}+\frac{\nu}{64 \alpha_{1}^{2} d^{2} M_{0}^{2}}\left\|\bar{D}_{\Delta t} \mathbf{E}_{h}^{n}\right\|_{0}^{2} \\
& \leq p_{1}\left(M_{0}\right)\left(\frac{1}{2}\left\|\mathbf{e}_{h}^{n-1}\right\|_{0}^{2}+\frac{1}{2}\left\|\mathbf{E}_{h}^{n-1}\right\|_{0}^{2}+\frac{\nu \varepsilon}{64 \alpha_{1}^{2} d^{2} M_{0}^{2}}\left|\mathbf{E}_{h}^{n-1}\right|_{1}^{2}+\frac{1}{2}\left\|\mathbf{E}_{h}^{n}\right\|_{0}^{2}\right) \\
& \quad+p_{2}\left(M_{0}\right)\left[\Delta t\|(\mathbf{u}, \mathbf{C})\|_{Z^{2}\left(t^{n-1}, t^{n}\right)}^{2}+h^{2}\left(\frac{1}{\Delta t}\|(\mathbf{u}, p, \mathbf{C})\|_{H^{1}\left(t^{n-1}, t^{n} ; \mathbb{H}^{2}\right)}^{2}+1\right)\right],
\end{aligned}
$$

where $p_{1}(\xi)=p_{1}(\xi ; \nu, \varepsilon)$ and $p_{2}(\xi)=p_{2}(\xi ; \nu)$ are polynomials in $\xi$ defined by

$$
\begin{array}{ll}
p_{1}: & c_{w, s}\left[\frac{1}{\nu}\left(\xi^{2}+1\right)+\left(1+\frac{\nu}{\xi^{2}}\right)\left(\xi^{4}+\xi^{2}+1\right)+\left(1+\frac{\xi^{2}}{\nu}\right)+\frac{1}{\varepsilon}\right] \leq \frac{c_{w, s}}{\nu \varepsilon}\left(\xi^{4}+4 \xi^{2}+6\right)=: p_{1}(\xi ; \nu, \varepsilon), \\
p_{2}: & c_{w, s}\left[\frac{1}{\nu^{2}}\left(\xi^{2}+1\right)+\left(1+\frac{\nu}{\xi^{2}}\right)\left(\xi^{4}+\xi^{2}+1\right)\right] \leq \frac{c_{w, s}}{\nu^{2}}\left(\xi^{4}+3 \xi^{2}+4\right)=: p_{2}(\xi ; \nu) .
\end{array}
$$

In the inequalities above the assumptions $\nu, \varepsilon \in(0,1]$ and $M_{0} \geq 1$ have been employed. By taking $c_{1}=p_{1}\left(M_{0} ; \nu, \varepsilon\right)$ and $c_{2}=p_{2}\left(M_{0} ; \nu\right)$ we finally obtain $(21)$.

\subsection{Proof of Theorem 1}

We prove Theorem 1 through three steps, where the function $D(h)$ defined in (13) is often used. 
Step 1 (Setting $c_{0}$ and $\left.h_{0}\right)$ : From (8) and (19) we have

$$
\left\|\mathbf{e}_{h}^{0}\right\|_{0} \leq\left\|\mathbf{u}_{h}^{0}-\mathbf{u}^{0}\right\|_{1}+\left\|\mathbf{u}^{0}-\hat{\mathbf{u}}_{h}^{0}\right\|_{1} \leq 2 \frac{\alpha_{31}}{\nu} h\left\|(u, p)^{0}\right\|_{H^{2} \times H^{1}}=\sqrt{2} c_{I} h
$$

for $c_{I}:=\left(\sqrt{2} \alpha_{31} / \nu\right)\left\|(u, p)^{0}\right\|_{H^{2} \times H^{1}}$. The constants $c_{1}$ and $c_{2}$ in Proposition 3 depend on $M_{0}$. Now, we take $M_{0}=\|\mathbf{C}\|_{C\left(L^{\infty}\right)}+1$. Then, $c_{1}$ and $c_{2}$ are fixed. Let $c_{3}$ and $c_{*}$ be constants defined by

$$
c_{3}:=\exp \left(\frac{3 c_{1} T}{2}\right) \max \left\{\sqrt{c_{2}}\|(\mathbf{u}, \mathbf{C})\|_{Z^{2}}, \sqrt{c_{2}}\left(\|(\mathbf{u}, p, \mathbf{C})\|_{H^{1}\left(\mathbb{H}^{2}\right)}+\sqrt{T}\right)+c_{I}\right\} .
$$

and $c_{*}:=c_{3}\left(8 \alpha_{1} d M_{0} / \sqrt{\nu \varepsilon}\right)$. We can choose sufficiently small positive constants $c_{0}$ and $h_{0}$ such that

$$
\begin{aligned}
\alpha_{21}\left[c_{*}\left\{c_{0}+h_{0} D\left(h_{0}\right)\right\}+\left(\alpha_{20}+\alpha_{32}\right) h_{0} D\left(h_{0}\right)\|\mathbf{C}\|_{C\left(H^{2}\right)}\right] & \leq 1, \\
(\Delta t \leq) \quad \frac{c_{0}}{D\left(h_{0}\right)} & \leq \frac{1}{2 c_{1}}, \\
\left(\Delta t|\mathbf{w}|_{1, \infty} \leq\right) \frac{c_{0}|\mathbf{w}|_{1, \infty}}{D\left(h_{0}\right)} & \leq \frac{1}{4},
\end{aligned}
$$

since $h D(h)$ and $1 / D(h)$ tend to zero as $h$ tends to zero.

Let $(h, \Delta t)$ be any pair satisfying (9). Since condition (4) is satisfied, Proposition 2 ensures the existence and uniqueness of the solution $\left(\mathbf{u}_{h}, p_{h}, \mathbf{C}_{h}\right)=\left\{\left(\mathbf{u}_{h}^{n}, p_{h}^{n}, \mathbf{C}_{h}^{n}\right)\right\}_{n=1}^{N_{T}} \subset V_{h} \times Q_{h} \times W_{h}$ of scheme (6) with (8).

Step 2 (Induction): By induction we show that the following property $\mathrm{P}(n)$ holds for $n \in\left\{0, \ldots, N_{T}\right\}$,

$$
\begin{aligned}
& \left(\text { (a) } \frac{1}{2}\left\|\mathbf{e}_{h}^{n}\right\|_{0}^{2}+\frac{1}{2}\left\|\mathbf{E}_{h}^{n}\right\|_{0}^{2}+\frac{\nu \varepsilon}{64 \alpha_{1}^{2} d^{2} M_{0}^{2}}\left|\mathbf{E}_{h}^{n}\right|_{1}^{2}+\frac{\nu}{2 \alpha_{1}^{2}}\left\|\mathbf{e}_{h}\right\|_{\ell_{n}^{2}\left(H^{1}\right)}^{2}+\delta_{0}\left|\epsilon_{h}\right|_{\ell_{n}^{2}\left(|\cdot|_{h}\right)}^{2}+\frac{\nu}{64 \alpha_{1}^{2} d^{2} M_{0}^{2}}\left\|\bar{D}_{\Delta t} \mathbf{E}_{h}\right\|_{\ell_{n}^{2}\left(L^{2}\right)}^{2}\right. \\
& \mathrm{P}(n):\left\{\begin{aligned}
\leq \exp \left(3 c_{1} n \Delta t\right)\left[\frac{1}{2}\left\|\mathbf{e}_{h}^{0}\right\|_{0}^{2}\right. & +\frac{1}{2}\left\|\mathbf{E}_{h}^{0}\right\|_{0}^{2}+\frac{\nu \varepsilon}{64 \alpha_{1}^{2} d^{2} M_{0}^{2}}\left|\mathbf{E}_{h}^{0}\right|_{1}^{2} \\
& \left.+c_{2}\left\{\Delta t^{2}\|(\mathbf{u}, \mathbf{C})\|_{Z^{2}\left(0, t^{n}\right)}^{2}+h^{2}\left(\|(\mathbf{u}, p, \mathbf{C})\|_{H^{1}\left(0, t^{n} ; \mathbb{H}^{2}\right)}^{2}+n \Delta t\right)\right\}\right],
\end{aligned}\right.
\end{aligned}
$$

where $\left\|\mathbf{e}_{h}\right\|_{\ell_{n}^{2}\left(H^{1}\right)}=\left|\epsilon_{h}\right|_{\ell_{n}^{2}\left(|\cdot|_{h}\right)}=\left\|\bar{D}_{\Delta t} \mathbf{E}_{h}\right\|_{\ell_{n}^{2}\left(L^{2}\right)}=0$ for $n=0$

$\mathrm{P}(n)-(\mathrm{a})$ can be rewritten as

$$
x_{n}+\Delta t \sum_{i=1}^{n} y_{i} \leq \exp \left(3 c_{1} n \Delta t\right)\left(x_{0}+\Delta t \sum_{i=1}^{n} b_{i}\right)
$$

where

$$
\begin{aligned}
x_{n} & :=\frac{1}{2}\left\|\mathbf{e}_{h}^{n}\right\|_{0}^{2}+\frac{1}{2}\left\|\mathbf{E}_{h}^{n}\right\|_{0}^{2}+\frac{\nu \varepsilon}{64 \alpha_{1}^{2} d^{2} M_{0}^{2}}\left|\mathbf{E}_{h}^{n}\right|_{1}^{2}, \quad y_{i}:=\frac{\nu}{2 \alpha_{1}^{2}}\left\|\mathbf{e}_{h}^{i}\right\|_{1}^{2}+\delta_{0}\left|\epsilon_{h}^{i}\right|_{h}^{2}+\frac{\nu}{64 \alpha_{1}^{2} d^{2} M_{0}^{2}}\left\|\bar{D}_{\Delta t} \mathbf{E}_{h}^{i}\right\|_{0}^{2} \\
b_{i} & :=c_{2}\left\{\Delta t\|(\mathbf{u}, \mathbf{C})\|_{Z^{2}\left(t^{i-1}, t^{i}\right)}^{2}+h^{2}\left(\frac{1}{\Delta t}\|(\mathbf{u}, p, \mathbf{C})\|_{H^{1}\left(t^{i-1}, t^{i} ; \mathbb{H}^{2}\right)}^{2}+1\right)\right\}
\end{aligned}
$$

We firstly prove the general step in the induction. Supposing that $\mathrm{P}(n-1)$ holds true for an integer $n \in\left\{1, \ldots, N_{T}\right\}$, we prove that $\mathrm{P}(n)$ also holds. We prove $\mathrm{P}(n)$-(a). Since (5) and (20) with $M_{0}=\|\mathbf{C}\|_{C\left(L^{\infty}\right)}+1(\geq 1)$ are satisfied from $(29 \mathrm{c})$ and $\mathrm{P}(n-1)-(\mathrm{b})$, respectively, we have (21) from Proposition 3 . The inequality (21) implies that

$$
\bar{D}_{\Delta t} x_{n}+y_{n} \leq c_{1}\left(x_{n}+x_{n-1}\right)+b_{n},
$$

which leads to

$$
x_{n}+\Delta t y_{n} \leq \exp \left(3 c_{1} \Delta t\right)\left(x_{n-1}+\Delta t b_{n}\right)
$$

by $\left(1+c_{1} \Delta t\right) /\left(1-c_{1} \Delta t\right) \leq\left(1+c_{1} \Delta t\right)\left(1+2 c_{1} \Delta t\right) \leq \exp \left(3 c_{1} \Delta t\right)$, where $c_{1} \Delta t \leq 1 / 2$ from (29b). From (31) and $\mathrm{P}(n-1)$-(a) we have

$$
x_{n}+\Delta t \sum_{i=1}^{n} y_{i} \leq \exp \left(3 c_{1} \Delta t\right)\left(x_{n-1}+\Delta t b_{n}\right)+\Delta t \sum_{i=1}^{n-1} y_{i} \leq \exp \left(3 c_{1} \Delta t\right)\left(x_{n-1}+\Delta t \sum_{i=1}^{n-1} y_{i}+\Delta t b_{n}\right)
$$




$$
\begin{aligned}
& \leq \exp \left(3 c_{1} \Delta t\right)\left[\exp \left\{3 c_{1}(n-1) \Delta t\right\}\left(x_{0}+\Delta t \sum_{i=1}^{n-1} b_{i}\right)+\Delta t b_{n}\right] \\
& \leq \exp \left(3 c_{1} n \Delta t\right)\left(x_{0}+\Delta t \sum_{i=1}^{n} b_{i}\right) .
\end{aligned}
$$

Thus, we obtain $\mathrm{P}(n)-(\mathrm{a})$.

For the proof of $\mathrm{P}(n)-(\mathrm{b})$ we prepare the estimate of $\left\|\mathbf{E}_{h}^{n}\right\|_{1}$. We have

$$
x_{0}=\frac{1}{2}\left\|\mathbf{e}_{h}^{0}\right\|_{0}^{2}+\frac{1}{2}\left\|\mathbf{E}_{h}^{0}\right\|_{0}^{2}+\frac{\nu \varepsilon}{64 \alpha_{1}^{2} d^{2} M_{0}^{2}}\left|\mathbf{E}_{h}^{0}\right|_{1}^{2}=\frac{1}{2}\left\|\mathbf{e}_{h}^{0}\right\|_{0}^{2} \leq c_{I}^{2} h^{2}
$$

from (27). $\mathrm{P}(n)-(\mathrm{a})$ with (32) implies that

$$
\begin{aligned}
& \frac{1}{2}\left\|\mathbf{e}_{h}^{n}\right\|_{0}^{2}+\frac{1}{2}\left\|\mathbf{E}_{h}^{n}\right\|_{0}^{2}+\frac{\nu \varepsilon}{64 \alpha_{1}^{2} d^{2} M_{0}^{2}}\left|\mathbf{E}_{h}^{n}\right|_{1}^{2}+\frac{\nu}{2 \alpha_{1}^{2}}\left\|\mathbf{e}_{h}\right\|_{\ell_{n}^{2}\left(H^{1}\right)}^{2}+\delta_{0}\left|\epsilon_{h}\right|_{\ell_{n}^{2}\left(\left.|\cdot|\right|_{h}\right)}^{2}+\frac{\nu}{64 \alpha_{1}^{2} d^{2} M_{0}^{2}}\left\|\bar{D}_{\Delta t} \mathbf{E}_{h}\right\|_{\ell_{n}^{2}\left(L^{2}\right)}^{2} \\
& \quad \leq \exp \left(3 c_{1} T\right)\left[c_{I}^{2} h^{2}+c_{2}\left\{\Delta t^{2}\|(\mathbf{u}, \mathbf{C})\|_{Z^{2}}^{2}+h^{2}\left(\|(\mathbf{u}, p, \mathbf{C})\|_{H^{1}\left(\mathbb{H}^{2}\right)}^{2}+T\right)\right\}\right] \\
& \quad \leq \exp \left(3 c_{1} T\right)\left[c_{2} \Delta t^{2}\|(\mathbf{u}, \mathbf{C})\|_{Z^{2}}^{2}+h^{2}\left\{c_{2}\left(\|(\mathbf{u}, p, \mathbf{C})\|_{H^{1}\left(\mathbb{H}^{2}\right)}^{2}+T\right)+c_{I}^{2}\right\}\right] \\
& \quad \leq\left\{c_{3}(\Delta t+h)\right\}^{2},
\end{aligned}
$$

which yields

$$
\left\|\mathbf{E}_{h}^{n}\right\|_{1} \leq \frac{8 \alpha_{1} d M_{0}}{\sqrt{\nu \varepsilon}} c_{3}(\Delta t+h)=c_{*}(\Delta t+h)
$$

from $\nu \varepsilon /\left(64 \alpha_{1}^{2} d^{2} M_{0}^{2}\right) \leq 1 /\left(64 d^{2}\right)<1 / 2$.

We prove $\mathrm{P}(n)-(\mathrm{b})$ as follows:

$$
\begin{aligned}
\left\|\mathbf{C}_{h}^{n}\right\|_{0, \infty} & \leq\left\|\mathbf{C}_{h}^{n}-\Pi_{h} \mathbf{C}^{n}\right\|_{0, \infty}+\left\|\Pi_{h} \mathbf{C}^{n}\right\|_{0, \infty} \leq \alpha_{21} D(h)\left\|\mathbf{C}_{h}^{n}-\Pi_{h} \mathbf{C}^{n}\right\|_{1}+\left\|\Pi_{h} \mathbf{C}^{n}\right\|_{0, \infty} \\
& \leq \alpha_{21} D(h)\left(\left\|\mathbf{C}_{h}^{n}-\hat{\mathbf{C}}_{h}^{n}\right\|_{1}+\left\|\hat{\mathbf{C}}_{h}^{n}-\mathbf{C}^{n}\right\|_{1}+\left\|\mathbf{C}^{n}-\Pi_{h} \mathbf{C}^{n}\right\|_{1}\right)+\left\|\Pi_{h} \mathbf{C}^{n}\right\|_{0, \infty} \\
& \leq \alpha_{21} D(h)\left[c_{*}(\Delta t+h)+\alpha_{32} h\left\|\mathbf{C}^{n}\right\|_{2}+\alpha_{20} h\left\|\mathbf{C}^{n}\right\|_{2}\right]+\left\|\mathbf{C}^{n}\right\|_{0, \infty} \\
& \leq \alpha_{21}\left[c_{*}\left\{c_{0}+h_{0} D\left(h_{0}\right)\right\}+\left(\alpha_{20}+\alpha_{32}\right) h_{0} D\left(h_{0}\right)\|\mathbf{C}\|_{C\left(H^{2}\right)}\right]+\|\mathbf{C}\|_{C\left(L^{\infty}\right)} \\
& \leq 1+\|\mathbf{C}\|_{C\left(L^{\infty}\right)},
\end{aligned}
$$

from (34), (9) and (29a). Therefore, $\mathrm{P}(n)$ holds true.

The proof of $\mathrm{P}(0)$ is easier than that of the general step. $\mathrm{P}(0)-(\mathrm{a})$ obviously holds with equality. $\mathrm{P}(0)-(\mathrm{b})$ is obtained as follows:

$$
\begin{aligned}
\left\|\mathbf{C}_{h}^{0}\right\|_{0, \infty} & \leq\left\|\mathbf{C}_{h}^{0}-\Pi_{h} \mathbf{C}^{0}\right\|_{0, \infty}+\left\|\Pi_{h} \mathbf{C}^{0}\right\|_{0, \infty} \leq \alpha_{21} D(h)\left(\left\|\mathbf{C}_{h}^{0}-\mathbf{C}^{0}\right\|_{1}+\left\|\mathbf{C}^{0}-\Pi_{h} \mathbf{C}^{0}\right\|_{1}\right)+\left\|\Pi_{h} \mathbf{C}^{0}\right\|_{0, \infty} \\
& \leq \alpha_{21}\left(\alpha_{20}+\alpha_{32}\right) h D(h)\left\|\mathbf{C}^{0}\right\|_{2}+\left\|\mathbf{C}^{0}\right\|_{0, \infty} \\
& \leq 1+\|\mathbf{C}\|_{C\left(L^{\infty}\right)} .
\end{aligned}
$$

Thus, the induction is completed.

Step 3: Finally we derive (10) and (11). Since $\mathrm{P}\left(N_{T}\right)$ holds true, we have (10) and

$$
\left\|\mathbf{e}_{h}\right\|_{\ell^{\infty}\left(L^{2}\right) \cap \ell^{2}\left(H^{1}\right)}, \quad\left|\epsilon_{h}\right|_{\ell^{2}\left(|\cdot|_{h}\right)}, \quad\left\|\bar{D}_{\Delta t} \mathbf{E}_{h}\right\|_{\ell^{2}\left(L^{2}\right)} \leq c_{\nu, \varepsilon} c_{w, s}(\Delta t+h)
$$

from (33). Combining (36) and the estimates

$$
\begin{aligned}
\left\|\mathbf{u}_{h}-\mathbf{u}\right\|_{\ell^{\infty}\left(L^{2}\right)} & \leq\left\|\mathbf{e}_{h}\right\|_{\ell^{\infty}\left(L^{2}\right)}+\|\boldsymbol{\eta}\|_{\ell^{\infty}\left(L^{2}\right)} \leq\left\|\mathbf{e}_{h}\right\|_{\ell^{\infty}\left(L^{2}\right)}+\frac{\alpha_{31}}{\nu} h\|(\mathbf{u}, p)\|_{C\left(H^{2} \times H^{1}\right)}, \\
\left\|\bar{D}_{\Delta t} \mathbf{C}_{h}^{n}-\frac{\partial \mathbf{C}^{n}}{\partial t}\right\|_{0} & \leq\left\|\bar{D}_{\Delta t} \mathbf{E}_{h}^{n}\right\|_{0}+\left\|\bar{D}_{\Delta t} \mathbf{\Xi}^{n}\right\|_{0}+\left\|\bar{D}_{\Delta t} \mathbf{C}^{n}-\frac{\partial \mathbf{C}^{n}}{\partial t}\right\|_{0} \\
& \leq\left\|\bar{D}_{\Delta t} \mathbf{E}_{h}^{n}\right\|_{0}+\frac{\alpha_{32} h}{\sqrt{\Delta t}}\|\mathbf{C}\|_{H^{1}\left(t^{n-1}, t^{n} ; H^{2}\right)}+\sqrt{\frac{\Delta t}{3}}\left\|\frac{\partial^{2} \mathbf{C}}{\partial t^{2}}\right\|_{L^{2}\left(t^{n-1}, t^{n} ; L^{2}\right)}
\end{aligned}
$$

we can obtain the first and the last inequalities of (11) with a positive constant $c_{\dagger}$ independent of $h$ and $\Delta t$. The other inequalities of (11) are similarly proved by using (34) and (36). 
Remark 8. We note that the error constant behaves like $\mathcal{O}(\exp [c T /(\nu \varepsilon)])(\nu, \varepsilon \downarrow 0)$ with respect to the viscosity $\nu$ and the elastic diffusion coefficient $\varepsilon$, since the main contribution is the exponential part of $c_{3}$ in (28), i.e., $\exp \left[3 c_{1} T / 2\right]=\exp \left[3 p_{1}\left(\|\mathbf{C}\|_{C\left(L^{\infty}\right)}+1 ; \nu, \varepsilon\right) T / 2\right]=\mathcal{O}(\exp [c T /(\nu \varepsilon)])$, where $(26)$ is used for the last equality. Although the dependency on $\nu$ and $\varepsilon$ of the coefficient is not good, it seems hard to avoid it. Similar coefficient $\mathcal{O}(\exp [c T / \nu])$ appears in the estimate of the Navier-Stokes equations, [9, 51]. As for the estimate independent of $\nu$, we refer to [40] for the Stokes equations and to [16] for the Oseen equations.

\subsection{A lemma for the proof of Theorem 2}

In the proof of Theorem 2 we use the next lemma.

Lemma 7. Suppose that Hypotheses 1 and 2 and the inequalities (10) and (11) hold. Let $m \in\left\{1, \ldots, N_{T}\right\}$ be any fixed number. Then, under the condition (5) we have the following.

$$
\begin{aligned}
& \Delta t \sum_{n=1}^{m}\left\langle\mathbf{r}_{h 1}^{n}, \bar{D}_{\Delta t} \mathbf{e}_{h}^{n}\right\rangle, \quad \Delta t \sum_{n=1}^{m}\left\langle\mathbf{r}_{h 2}^{n}, \bar{D}_{\Delta t} \mathbf{e}_{h}^{n}\right\rangle \leq \frac{\Delta t}{6} \sum_{n=1}^{m}\left\|\bar{D}_{\Delta t} \mathbf{e}_{h}^{n}\right\|_{0}^{2}+c_{\nu, \varepsilon} c_{w, s}\left(\Delta t^{2}+h^{2}\right), \\
& \Delta t \sum_{n=1}^{m}\left\langle\mathbf{r}_{h 3}^{n}, \bar{D}_{\Delta t} \mathbf{e}_{h}^{n}\right\rangle, \quad \Delta t \sum_{n=1}^{m}\left\langle\mathbf{r}_{h 4}^{n}, \bar{D}_{\Delta t} \mathbf{e}_{h}^{n}\right\rangle \leq \frac{\nu}{4}\left\|\mathrm{D}\left(\mathbf{e}_{h}^{m}\right)\right\|_{0}^{2}+c_{\nu, \varepsilon} c_{w, s}\left(\Delta t^{2}+h^{2}\right) .
\end{aligned}
$$

Proof. The inequalities (37a) are obtained by combining (22a) and (22b) with

$$
\left\langle\mathbf{r}_{h i}^{n}, \bar{D}_{\Delta t} \mathbf{e}_{h}^{n}\right\rangle \leq\left\|\mathbf{r}_{h i}^{n}\right\|_{0}\left\|\bar{D}_{\Delta t} \mathbf{e}_{h}^{n}\right\|_{0} \leq \frac{3}{2}\left\|\mathbf{r}_{h i}^{n}\right\|_{0}^{2}+\frac{1}{6}\left\|\bar{D}_{\Delta t} \mathbf{e}_{h}^{n}\right\|_{0}^{2}, \quad i=1,2 .
$$

We prove (37b). For $i=3,4$ we have

$$
\begin{aligned}
& \Delta t \sum_{n=1}^{m}\left\langle\mathbf{r}_{h i}^{n}, \bar{D}_{\Delta t} \mathbf{e}_{h}^{n}\right\rangle=\sum_{n=1}^{m}\left(\mathbf{r}_{h i}^{n}, \nabla \mathbf{e}_{h}^{n}-\nabla \mathbf{e}_{h}^{n-1}\right)=\left(\mathbf{r}_{h i}^{m}, \nabla \mathbf{e}_{h}^{m}\right)-\sum_{n=1}^{m-1}\left(\mathbf{r}_{h i}^{n+1}-\mathbf{r}_{h i}^{n}, \nabla \mathbf{e}_{h}^{n}\right)-\left(\mathbf{r}_{h i}^{1}, \nabla \mathbf{e}_{h}^{0}\right) \\
& \quad \leq \alpha_{1}\left\|\mathbf{r}_{h i}^{m}\right\|_{-1}\left\|\mathrm{D}\left(\mathbf{e}_{h}^{m}\right)\right\|_{0}+\sum_{n=1}^{m-1}\left\|\mathbf{r}_{h i}^{n+1}-\mathbf{r}_{h i}^{n}\right\|_{0}\left\|\mathbf{e}_{h}^{n}\right\|_{1}+\left\|\mathbf{r}_{h i}^{1}\right\|_{-1}\left\|\mathbf{e}_{h}^{0}\right\|_{1} \\
& \quad \leq \frac{\nu}{4}\left\|\mathrm{D}\left(\mathbf{e}_{h}^{m}\right)\right\|_{0}^{2}+\frac{\alpha_{1}^{2}}{\nu}\left\|\mathbf{r}_{h i}^{m}\right\|_{-1}^{2}+\alpha_{1} \sum_{n=1}^{m-1}\left\|\mathbf{r}_{h i}^{n+1}-\mathbf{r}_{h i}^{n}\right\|_{0}\left\|\mathrm{D}\left(\mathbf{e}_{h}^{n}\right)\right\|_{0}+\frac{1}{2}\left\|\mathbf{r}_{h i}^{1}\right\|_{-1}^{2}+\frac{1}{2}\left\|\mathbf{e}_{h}^{0}\right\|_{1}^{2} \\
& \left.\quad \leq \frac{\nu}{4}\left\|\mathrm{D}\left(\mathbf{e}_{h}^{m}\right)\right\|_{0}^{2}+\alpha_{1} \sum_{n=1}^{m-1}\left\|\mathbf{r}_{h i}^{n+1}-\mathbf{r}_{h i}^{n}\right\|_{0}\left\|\mathrm{D}\left(\mathbf{e}_{h}^{n}\right)\right\|_{0}+c_{\nu, \varepsilon} c_{w, s}\left(\Delta t^{2}+h^{2}\right) \quad \text { (by }(22 \mathrm{c}),(22 \mathrm{~d}),(27), \mathrm{Thm} .1\right)
\end{aligned}
$$

Applying Hölder's inequality, we have

$$
\begin{aligned}
& \| \operatorname{tr} \mathbf{C}^{n+1}\left(\mathbf{C}^{n+1}-\mathbf{C}^{n}\right)-\operatorname{tr} \mathbf{C}^{n}\left(\mathbf{C}^{n}-\mathbf{C}^{n-1}\right)\left\|_{0}=\right\| \int_{t^{n}}^{t^{n+1}} \frac{\partial}{\partial t}\{\operatorname{tr} \mathbf{C}(t)[\mathbf{C}(t)-\mathbf{C}(t-\Delta t)]\} d t \|_{0} \\
& \leq\left\|\int_{t^{n}}^{t^{n+1}} \operatorname{tr} \frac{\partial \mathbf{C}}{\partial t}(t)[\mathbf{C}(t)-\mathbf{C}(t-\Delta t)] d t\right\|_{0}+\left\|\int_{t^{n}}^{t^{n+1}} \operatorname{tr} \mathbf{C}(t)\left[\frac{\partial \mathbf{C}}{\partial t}(t)-\frac{\partial \mathbf{C}}{\partial t}(t-\Delta t)\right] d t\right\|_{0} \\
&=\left\|\int_{t^{n}}^{t^{n+1}} \operatorname{tr} \frac{\partial \mathbf{C}}{\partial t}(t) d t \int_{t-\Delta t}^{t} \frac{\partial \mathbf{C}}{\partial t}(s) d s\right\|_{0}+\left\|\int_{t^{n}}^{t^{n+1}} \operatorname{tr} \mathbf{C}(t) d t \int_{t-\Delta t}^{t} \frac{\partial^{2} \mathbf{C}}{\partial t^{2}}(s) d s\right\|_{0} \\
& \leq \int_{t^{n}}^{t^{n+1}}\left\|\operatorname{tr} \frac{\partial \mathbf{C}}{\partial t}(t)\right\|_{0,4} d t \int_{t^{n-1}}^{t^{n+1}}\left\|\frac{\partial \mathbf{C}}{\partial t}(s)\right\|_{0,4} d s+\int_{t^{n}}^{t^{n+1}}\|\operatorname{tr} \mathbf{C}(t)\|_{0, \infty} d t \int_{t^{n-1}}^{t^{n+1}}\left\|\frac{\partial^{2} \mathbf{C}}{\partial t^{2}}(s)\right\| \|_{0} d s \\
& \leq \Delta t^{3 / 4}\left(\int_{t^{n}}^{t^{n+1}}\left\|\frac{\partial \mathbf{C}}{\partial t}(t)\right\|_{0,4}^{4} d t\right)^{1 / 4}(2 \Delta t)^{3 / 4}\left(\int_{t^{n-1}}^{t^{n+1}}\left\|\frac{\partial \mathbf{C}}{\partial t}(s)\right\|_{0,4}^{4} d s\right)^{1 / 4}+d \Delta t\|\mathbf{C}\|_{C\left(L^{\infty}\right)} \sqrt{2 \Delta t}\|\mathbf{C}\|_{H^{2}\left(t^{n-1}, t^{n+1} ; L^{2}\right)} \\
& \leq c_{s} \Delta t^{3 / 2}\left(\left\|\frac{\partial \mathbf{C}}{\partial t}\right\|_{L^{4}\left(t^{n-1}, t^{n+1} ; L^{4}\right)}^{2}+\|\mathbf{C}\|_{H^{2}\left(t^{n-1}, t^{n+1} ; L^{2}\right)}\right), \\
&\left\|\operatorname{tr} \mathbf{C}^{n+1} \mathbf{\Xi}^{n}-\operatorname{tr} \mathbf{C}^{n} \mathbf{\Xi}^{n-1}\right\|_{0} \leq\left\|\left(\operatorname{tr} \mathbf{C}^{n+1}-\operatorname{tr} \mathbf{C}^{n}\right) \boldsymbol{\Xi}^{n}\right\|_{0}+\left\|\operatorname{tr} \mathbf{C}^{n}\left(\mathbf{\Xi}^{n}-\mathbf{\Xi}^{n-1}\right)\right\|_{0}
\end{aligned}
$$




$$
\begin{aligned}
& \leq\left\|\operatorname{tr} \mathbf{C}^{n+1}-\operatorname{tr} \mathbf{C}^{n}\right\|_{0,3}\left\|\boldsymbol{\Xi}^{n}\right\|_{0,6}+\left\|\operatorname{tr} \mathbf{C}^{n}\right\|_{0,3}\left\|\boldsymbol{\Xi}^{n}-\boldsymbol{\Xi}^{n-1}\right\|_{0,6} \\
& \leq \sqrt{\Delta t}\left\|\frac{\partial(\operatorname{tr} \mathbf{C})}{\partial t}\right\|_{L^{2}\left(t^{n}, t^{n+1} ; L^{3}\right)}\left\|\boldsymbol{\Xi}^{n}\right\|_{0,6}+\left\|\mathbf{C}^{n}\right\|_{1} \sqrt{\Delta t}\left\|\frac{\partial \boldsymbol{\Xi}}{\partial t}\right\|_{L^{2}\left(t^{n-1}, t^{n} ; L^{6}\right)} \\
& \leq c \sqrt{\Delta t}\left(\|\mathbf{C}\|_{H^{1}\left(t^{n}, t^{n+1} ; H^{1}\right)}\left\|\boldsymbol{\Xi}^{n}\right\|_{1}+\left\|\mathbf{C}^{n}\right\|_{1}\|\boldsymbol{\Xi}\|_{H^{1}\left(t^{n-1}, t^{n} ; H^{1}\right)}\right) \\
& \leq c \sqrt{\Delta t}\left(\|\mathbf{C}\|_{H^{1}\left(t^{n}, t^{n+1} ; H^{1}\right)} \alpha_{32} h\left\|\mathbf{C}^{n}\right\|_{2}+\left\|\mathbf{C}^{n}\right\|_{1} \alpha_{32} h\|\mathbf{C}\|_{H^{1}\left(t^{n-1}, t^{n} ; H^{2}\right)}\right) \\
& \leq c_{s} h \sqrt{\Delta t}\|\mathbf{C}\|_{H^{1}\left(t^{n-1}, t^{n+1} ; H^{2}\right)}, \\
& \left\|\operatorname{tr} \mathbf{C}^{n+1} \mathbf{E}_{h}^{n}-\operatorname{tr} \mathbf{C}^{n} \mathbf{E}_{h}^{n-1}\right\|_{0} \leq\left\|\left(\operatorname{tr} \mathbf{C}^{n+1}-\operatorname{tr} \mathbf{C}^{n}\right) \mathbf{E}_{h}^{n}\right\|_{0}+\left\|\operatorname{tr} \mathbf{C}^{n}\left(\mathbf{E}_{h}^{n}-\mathbf{E}_{h}^{n-1}\right)\right\|_{0} \\
& \leq\left\|\operatorname{tr} \mathbf{C}^{n+1}-\operatorname{tr} \mathbf{C}^{n}\right\|_{0,3}\left\|\mathbf{E}_{h}^{n}\right\|_{0,6}+\left\|\operatorname{tr} \mathbf{C}^{n}\right\|_{0, \infty}\left\|\mathbf{E}_{h}^{n}-\mathbf{E}_{h}^{n-1}\right\|_{0} \\
& \leq \sqrt{\Delta t}\left\|\frac{\partial(\operatorname{tr} \mathbf{C})}{\partial t}\right\|_{L^{2}\left(t^{n}, t^{n+1} ; L^{3}\right)}\left\|\mathbf{E}_{h}^{n}\right\|_{1}+\left\|\operatorname{tr} \mathbf{C}^{n}\right\|_{0, \infty} \Delta t\left\|\bar{D}_{\Delta t} \mathbf{E}_{h}^{n}\right\|_{0} \\
& \leq c_{\nu, \varepsilon} c_{w, s} \sqrt{\Delta t}\left[(\Delta t+h)\|\mathbf{C}\|_{H^{1}\left(t^{n}, t^{n+1} ; H^{1}\right)}+\sqrt{\Delta t}\left\|\bar{D}_{\Delta t} \mathbf{E}_{h}^{n}\right\|_{0}\right] \quad \text { (by Thm.1). } \\
& \left\|\mathbf{r}_{h 3}^{n+1}-\mathbf{r}_{h 3}^{n}\right\|_{0}=\left\|\left(\operatorname{tr} \mathbf{C}^{n+1}\right)\left(\mathbf{C}^{n+1}-\mathbf{C}^{n}+\mathbf{\Xi}^{n}-\mathbf{E}_{h}^{n}\right)-\left(\operatorname{tr} \mathbf{C}^{n}\right)\left(\mathbf{C}^{n}-\mathbf{C}^{n-1}+\mathbf{\Xi}^{n-1}-\mathbf{E}_{h}^{n-1}\right)\right\|_{0} \\
& \leq\left\|\left(\operatorname{tr} \mathbf{C}^{n+1}\right)\left(\mathbf{C}^{n+1}-\mathbf{C}^{n}\right)-\left(\operatorname{tr} \mathbf{C}^{n}\right)\left(\mathbf{C}^{n}-\mathbf{C}^{n-1}\right)\right\|_{0}+\left\|\left(\operatorname{tr} \mathbf{C}^{n+1}\right) \boldsymbol{\Xi}^{n}-\left(\operatorname{tr} \mathbf{C}^{n}\right) \boldsymbol{\Xi}^{n-1}\right\|_{0} \\
& +\left\|\left(\operatorname{tr} \mathbf{C}^{n+1}\right) \mathbf{E}_{h}^{n}-\left(\operatorname{tr} \mathbf{C}^{n}\right) \mathbf{E}_{h}^{n-1}\right\|_{0} \\
& \leq c_{\nu, \varepsilon} c_{w, s} \sqrt{\Delta t}\left[\Delta t\left\|\frac{\partial \mathbf{C}}{\partial t}\right\|_{L^{4}\left(t^{n-1}, t^{n+1} ; L^{4}\right)}^{2}+\Delta t\|\mathbf{C}\|_{H^{2}\left(t^{n-1}, t^{n+1} ; L^{2}\right)}+(\Delta t+h)\|\mathbf{C}\|_{H^{1}\left(t^{n-1}, t^{n+1} ; H^{2}\right)}+\sqrt{\Delta t}\left\|\bar{D}_{\Delta t} \mathbf{E}_{h}^{n}\right\|_{0}\right] .
\end{aligned}
$$

Combining (39) with (38) with $i=3$, we get

$$
\begin{aligned}
& \begin{array}{l}
\Delta t \sum_{n=1}^{m}\left\langle\mathbf{r}_{h 3}^{n}, \bar{D}_{\Delta t} \mathbf{e}_{h}^{n}\right\rangle \\
\leq \frac{\nu}{4}\left\|\mathrm{D}\left(\mathbf{e}_{h}^{m}\right)\right\|_{0}^{2}+c_{\nu, \varepsilon} c_{w, s}\left\{\left(\Delta t^{2}+h^{2}\right)+\sum_{n=1}^{m-1}\left[\Delta t\left\|\frac{\partial \mathbf{C}}{\partial t}\right\|_{L^{4}\left(t^{n-1}, t^{n+1} ; L^{4}\right)}^{2}+\Delta t\|\mathbf{C}\|_{H^{2}\left(t^{n-1}, t^{n+1} ; L^{2}\right)}\right.\right. \\
\left.\left.\quad+(\Delta t+h)\|\mathbf{C}\|_{H^{1}\left(t^{n-1}, t^{n+1} ; H^{2}\right)}+\sqrt{\Delta t}\left\|\bar{D}_{\Delta t} \mathbf{E}_{h}^{n}\right\|_{0}\right] \sqrt{\Delta t}\left\|\mathrm{D}\left(\mathbf{e}_{h}^{n}\right)\right\|_{0}\right\}
\end{array} \\
& \begin{array}{r}
\leq \frac{\nu}{4}\left\|\mathrm{D}\left(\mathbf{e}_{h}^{m}\right)\right\|_{0}^{2}+c_{\nu, \varepsilon} c_{w, s}\left\{\left(\Delta t^{2}+h^{2}\right)+\left\|\mathbf{e}_{h}\right\|_{\ell^{2}\left(H^{1}\right)}^{2}+\left\|\bar{D}_{\Delta t} \mathbf{E}_{h}\right\|_{\ell^{2}\left(L^{2}\right)}^{2}+2\left(\Delta t^{2}+h^{2}\right)\|\mathbf{C}\|_{H^{1}\left(H^{2}\right)}^{2}\right. \\
\left.+\Delta t^{2}\left(\left\|\frac{\partial \mathbf{C}}{\partial t}\right\|_{L^{4}\left(L^{4}\right)}^{4}+\|\mathbf{C}\|_{H^{2}\left(L^{2}\right)}^{2}\right)\right\}
\end{array} \\
& \leq \frac{\nu}{4}\left\|\mathrm{D}\left(\mathbf{e}_{h}^{m}\right)\right\|_{0}^{2}+c_{\nu, \varepsilon}^{\prime} c_{w, s}^{\prime}\left(\Delta t^{2}+h^{2}\right),
\end{aligned}
$$

where in the last inequality we have employed Theorem 1 and the relation $\left[L^{2}\left(0, T ; H^{1}(\Omega)\right) \cap H^{1}\left(0, T ; L^{2}(\Omega)\right)\right] \hookrightarrow$ $L^{4}\left(0, T ; L^{4}(\Omega)\right)$ yielding the inequality $\|\partial \mathbf{C} / \partial t\|_{L^{4}\left(L^{4}\right)} \leq c\|\partial \mathbf{C} / \partial t\|_{L^{2}\left(H^{1}\right) \cap H^{1}\left(L^{2}\right)} \leq c\|\mathbf{C}\|_{H^{1}\left(H^{1}\right) \cap H^{2}\left(L^{2}\right)} \leq c_{s}$. Thus, the first inequality of (37b) is proved. We prove the other inequality of (37b). For $\left\|\mathbf{r}_{h 4}^{n+1}-\mathbf{r}_{h 4}^{n}\right\|_{0}$ we have

$$
\begin{aligned}
& \left\|\mathbf{r}_{h 4}^{n+1}-\mathbf{r}_{h 4}^{n}\right\|_{0}=\left\|\left[\operatorname{tr}\left(\boldsymbol{\Xi}^{n+1}-\mathbf{E}_{h}^{n+1}\right)\right] \mathbf{C}_{h}^{n}-\left[\operatorname{tr}\left(\boldsymbol{\Xi}^{n}-\mathbf{E}_{h}^{n}\right)\right] \mathbf{C}_{h}^{n}+\left[\operatorname{tr}\left(\boldsymbol{\Xi}^{n}-\mathbf{E}_{h}^{n}\right)\right] \mathbf{C}_{h}^{n}-\left[\operatorname{tr}\left(\boldsymbol{\Xi}^{n}-\mathbf{E}_{h}^{n}\right)\right] \mathbf{C}_{h}^{n-1}\right\|_{0} \\
& =\left\|\left[\operatorname{tr}\left(\boldsymbol{\Xi}^{n+1}-\boldsymbol{\Xi}^{n}\right)\right] \mathbf{C}_{h}^{n}-\left[\operatorname{tr}\left(\mathbf{E}_{h}^{n+1}-\mathbf{E}_{h}^{n}\right)\right] \mathbf{C}_{h}^{n}+\left[\operatorname{tr}\left(\boldsymbol{\Xi}^{n}-\mathbf{E}_{h}^{n}\right)\right]\left[\Delta t \bar{D}_{\Delta t} \mathbf{E}_{h}^{n}-\left(\boldsymbol{\Xi}^{n}-\boldsymbol{\Xi}^{n-1}\right)+\left(\mathbf{C}^{n}-\mathbf{C}^{n-1}\right)\right]\right\|_{0} \\
& \leq c\left[\left\|\mathbf{C}_{h}^{n}\right\|_{0, \infty}\left(\left\|\boldsymbol{\Xi}^{n+1}-\boldsymbol{\Xi}^{n}\right\|_{0}+\Delta t\left\|\bar{D}_{\Delta t} \mathbf{E}_{h}^{n+1}\right\|_{0}\right)+\left\|\boldsymbol{\Xi}^{n}-\mathbf{E}_{h}^{n}\right\|_{0, \infty}\left(\Delta t\left\|\bar{D}_{\Delta t} \mathbf{E}_{h}^{n}\right\|_{0}+\left\|\boldsymbol{\Xi}^{n}-\boldsymbol{\Xi}^{n-1}\right\|_{0}\right)\right. \\
& \left.\quad+\left(\left\|\boldsymbol{\Xi}^{n}\right\|_{0}+\left\|\mathbf{E}_{h}^{n}\right\|_{0}\right)\left\|\mathbf{C}^{n}-\mathbf{C}^{n-1}\right\|_{0, \infty}\right] \\
& \leq c\left[\left(2\|\mathbf{C}\|_{C\left(L^{\infty}\right)}+1\right)\left\{\sqrt{\Delta t}\left\|\boldsymbol{\Xi}_{H^{1}\left(t^{n}, t^{n+1} ; L^{2}\right)}+\Delta t\left(\left\|\bar{D}_{\Delta t} \mathbf{E}_{h}^{n+1}\right\|_{0}+\left\|\bar{D}_{\Delta t} \mathbf{E}_{h}^{n}\right\|_{0}\right)+\sqrt{\Delta t}\right\| \boldsymbol{\Xi}_{H^{1}\left(t^{n-1}, t^{n} ; L^{2}\right)}\right\}\right. \\
& \left.\quad+\left(\left\|\boldsymbol{\Xi}^{n}\right\|_{0}+\left\|\mathbf{E}_{h}^{n}\right\|_{0}\right) \sqrt{\Delta t}\|\mathbf{C}\|_{H^{1}\left(t^{n-1}, t^{n} ; L^{\infty}\right)}\right]
\end{aligned}
$$




$$
\begin{aligned}
& \leq c_{\nu, \varepsilon} c_{w, s} \sqrt{\Delta t}\left[\alpha_{32} h\|\mathbf{C}\|_{H^{1}\left(t^{n-1}, t^{n+1} ; H^{2}\right)}+\sqrt{\Delta t}\left(\left\|\bar{D}_{\Delta t} \mathbf{E}_{h}^{n+1}\right\|_{0}+\left\|\bar{D}_{\Delta t} \mathbf{E}_{h}^{n}\right\|_{0}\right)\right. \\
& \left.\quad+\left(\alpha_{32} h\left\|\mathbf{C}^{n}\right\|_{2}+\left\|\mathbf{E}_{h}^{n}\right\|_{0}\right)\|\mathbf{C}\|_{H^{1}\left(t^{n-1}, t^{n} ; H^{2}\right)}\right] \\
& \leq c_{\nu, \varepsilon}^{\prime} c_{w, s}^{\prime} \sqrt{\Delta t}\left[\sqrt{\Delta t}\left(\left\|\bar{D}_{\Delta t} \mathbf{E}_{h}^{n+1}\right\|_{0}+\left\|\bar{D}_{\Delta t} \mathbf{E}_{h}^{n}\right\|_{0}\right)+(\Delta t+h)\|\mathbf{C}\|_{H^{1}\left(t^{n-1}, t^{n+1} ; H^{2}\right)}\right]
\end{aligned}
$$

where we have used the estimates,

$$
\begin{aligned}
& \mathbf{C}_{h}^{n}-\mathbf{C}_{h}^{n-1}=\left(\mathbf{E}_{h}^{n}-\boldsymbol{\Xi}^{n}+\mathbf{C}^{n}\right)-\left(\mathbf{E}_{h}^{n-1}-\boldsymbol{\Xi}^{n-1}+\mathbf{C}^{n-1}\right)=\Delta t \bar{D}_{\Delta t} \mathbf{E}_{h}^{n}-\left(\boldsymbol{\Xi}^{n}-\boldsymbol{\Xi}^{n-1}\right)+\left(\mathbf{C}^{n}-\mathbf{C}^{n-1}\right), \\
& \left.\left\|\boldsymbol{\Xi}^{n}-\mathbf{E}_{h}^{n}\right\|_{0, \infty}=\left\|\mathbf{C}^{n}-\mathbf{C}_{h}^{n}\right\|_{0, \infty} \leq\left\|\mathbf{C}^{n}\right\|_{0, \infty}+\left\|\mathbf{C}_{h}^{n}\right\|_{0, \infty} \leq 2\|\mathbf{C}\|_{C\left(L^{\infty}\right)}+1 \quad \text { (by Thm. } 1\right) .
\end{aligned}
$$

Combining (41) with (38) with $i=4$, we have

$$
\begin{aligned}
\Delta t & \sum_{n=1}^{m}\left\langle\mathbf{r}_{h 4}^{n}, \bar{D}_{\Delta t} \mathbf{e}_{h}^{n}\right\rangle \\
\leq & \frac{\nu}{4}\left\|\mathrm{D}\left(\mathbf{e}_{h}^{m}\right)\right\|_{0}^{2}+c_{\nu, \varepsilon} c_{w, s}\left\{\left(\Delta t^{2}+h^{2}\right)\right. \\
& \left.\quad+\sum_{n=1}^{m-1}\left[\sqrt{\Delta t}\left(\left\|\bar{D}_{\Delta t} \mathbf{E}_{h}^{n+1}\right\|_{0}+\left\|\bar{D}_{\Delta t} \mathbf{E}_{h}^{n}\right\|_{0}\right)+(\Delta t+h)\|\mathbf{C}\|_{H^{1}\left(t^{n-1}, t^{n+1} ; H^{2}\right)}\right] \sqrt{\Delta t}\left\|\mathrm{D}\left(\mathbf{e}_{h}^{n}\right)\right\|_{0}\right\} \\
\leq & \frac{\nu}{4}\left\|\mathrm{D}\left(\mathbf{e}_{h}^{m}\right)\right\|_{0}^{2}+c_{\nu, \varepsilon} c_{w, s}\left\{\left(\Delta t^{2}+h^{2}\right)+\left\|\mathbf{e}_{h}\right\|_{\ell^{2}\left(H^{1}\right)}^{2}+\left(\Delta t^{2}+h^{2}\right)\|\mathbf{C}\|_{H^{1}\left(H^{2}\right)}^{2}+\left\|\bar{D}_{\Delta t} \mathbf{E}_{h}\right\|_{\ell^{2}\left(L^{2}\right)}^{2}\right\} \\
\leq & \frac{\nu}{4}\left\|\mathrm{D}\left(\mathbf{e}_{h}^{m}\right)\right\|_{0}^{2}+c_{\nu, \varepsilon}^{\prime} c_{w, s}^{\prime}\left(\Delta t^{2}+h^{2}\right) \quad(\text { by Thm.1), }
\end{aligned}
$$

which is the other inequality of (37b).

\subsection{Proof of Theorem 2}

Let $p_{h}^{0}:=\left[\Pi_{h}^{\mathrm{SP}}\left(\mathbf{u}^{0}, 0, \mathbf{C}^{0}\right)\right]_{2}$, which leads to $\left(\mathbf{u}_{h}^{0}, p_{h}^{0}, \mathbf{C}_{h}^{0}\right)=\left[\Pi_{h}^{\mathrm{SP}}\left(\mathbf{u}^{0}, 0, \mathbf{C}^{0}\right)\right]$. Substituting $\left(\bar{D}_{\Delta t} \mathbf{e}_{h}^{n}, 0\right) \in V_{h} \times Q_{h}$ into $\left(\mathbf{v}_{h}, q_{h}\right)$ in (17a) and using

$$
\frac{\mathbf{e}_{h}^{n}-\mathbf{e}_{h}^{n-1} \circ X_{1}^{n}}{\Delta t}=\bar{D}_{\Delta t} \mathbf{e}_{h}^{n}+\frac{\mathbf{e}_{h}^{n-1}-\mathbf{e}_{h}^{n-1} \circ X_{1}^{n}}{\Delta t}
$$

we have

$$
\left\|\bar{D}_{\Delta t} \mathbf{e}_{h}^{n}\right\|_{0}^{2}+\nu a_{u}\left(e_{h}^{n}, \bar{D}_{\Delta t} \mathbf{e}_{h}^{n}\right)+b\left(\bar{D}_{\Delta t} \mathbf{e}_{h}^{n}, \epsilon_{h}^{n}\right)=\left\langle\mathbf{r}_{h}^{n}, \bar{D}_{\Delta t} \mathbf{e}_{h}^{n}\right\rangle-\frac{1}{\Delta t}\left(\mathbf{e}_{h}^{n-1}-\mathbf{e}_{h}^{n-1} \circ X_{1}^{n}, \bar{D}_{\Delta t} \mathbf{e}_{h}^{n}\right) .
$$

On the other hand, setting $\mathbf{v}_{h}=\mathbf{0} \in V_{h}$ in (17a), we have for $n=1, \ldots, N_{T}$

$$
b\left(\mathbf{e}_{h}^{n}, q_{h}\right)-\mathcal{S}_{h}\left(\epsilon_{h}^{n}, q_{h}\right)=0, \quad \forall q_{h} \in Q_{h},
$$

From the definitions of $\left(\mathbf{u}_{h}^{0}, p_{h}^{0}, \mathbf{C}_{h}^{0}\right)$ and $\left(\hat{\mathbf{u}}_{h}^{0}, \hat{p}_{h}^{0}, \hat{\mathbf{C}}_{h}^{0}\right)$ we have

$$
b\left(\mathbf{e}_{h}^{0}, q_{h}\right)-\mathcal{S}_{h}\left(\epsilon_{h}^{0}, q_{h}\right)=b\left(\mathbf{u}_{h}^{0}, q_{h}\right)-\mathcal{S}_{h}\left(p_{h}^{0}, q_{h}\right)-\left\{b\left(\hat{\mathbf{u}}_{h}^{0}, q_{h}\right)-\mathcal{S}_{h}\left(\hat{p}_{h}^{0}, q_{h}\right)\right\}=b\left(\mathbf{u}^{0}, q_{h}\right)-b\left(\mathbf{u}^{0}, q_{h}\right)=0, \quad \forall q_{h} \in Q_{h},
$$

which implies that (44) holds also for $n=0$. Hence, we get for $n=1, \ldots, N_{T}$

$$
b\left(\bar{D}_{\Delta t} \mathbf{e}_{h}^{n}, q_{h}\right)-\mathcal{S}_{h}\left(\bar{D}_{\Delta t} \epsilon_{h}^{n}, q_{h}\right)=0, \quad \forall q_{h} \in Q_{h},
$$

which yields

$$
b\left(\bar{D}_{\Delta t} \mathbf{e}_{h}^{n}, \epsilon_{h}^{n}\right)-\mathcal{S}_{h}\left(\bar{D}_{\Delta t} \epsilon_{h}^{n}, \epsilon_{h}^{n}\right)=0
$$

by setting $q_{h}=\epsilon_{h}^{n} \in Q_{h}$. Subtracting (45) from (43), we have for $n=1, \ldots, N_{T}$

$$
\left\|\bar{D}_{\Delta t} \mathbf{e}_{h}^{n}\right\|_{0}^{2}+\nu a_{u}\left(e_{h}^{n}, \bar{D}_{\Delta t} \mathbf{e}_{h}^{n}\right)+\mathcal{S}_{h}\left(\bar{D}_{\Delta t} \epsilon_{h}^{n}, \epsilon_{h}^{n}\right)=\left\langle\mathbf{r}_{h}^{n}, \bar{D}_{\Delta t} \mathbf{e}_{h}^{n}\right\rangle-\left(\frac{\mathbf{e}_{h}^{n-1}-\mathbf{e}_{h}^{n-1} \circ X_{1}^{n}}{\Delta t}, \bar{D}_{\Delta t} \mathbf{e}_{h}^{n}\right) .
$$


From the estimates,

$$
\begin{aligned}
\nu a_{u}\left(\mathbf{e}_{h}^{n}, \bar{D}_{\Delta t} \mathbf{e}_{h}^{n}\right) & =\bar{D}_{\Delta t}\left(\frac{\nu}{2} a_{u}\left(\mathbf{e}_{h}^{n}, \mathbf{e}_{h}^{n}\right)\right)+\frac{\nu \Delta t}{2} a_{u}\left(\bar{D}_{\Delta t} \mathbf{e}_{h}^{n}, \bar{D}_{\Delta t} \mathbf{e}_{h}^{n}\right) \geq \bar{D}_{\Delta t}\left(\nu\left\|\mathrm{D}\left(\mathbf{e}_{h}^{n}\right)\right\|_{0}^{2}\right), \\
\mathcal{S}_{h}\left(\bar{D}_{\Delta t} \epsilon_{h}^{n}, \epsilon_{h}^{n}\right) & =\bar{D}_{\Delta t}\left(\frac{1}{2} \mathcal{S}_{h}\left(\epsilon_{h}^{n}, \epsilon_{h}^{n}\right)\right)+\frac{\Delta t}{2} \mathcal{S}_{h}\left(\bar{D}_{\Delta t} \epsilon_{h}^{n}, \bar{D}_{\Delta t} \epsilon_{h}^{n}\right) \geq \bar{D}_{\Delta t}\left(\frac{\delta_{0}}{2}\left|\epsilon_{h}^{n}\right|_{h}^{2}\right), \\
\left|\frac{1}{\Delta t}\left(\mathbf{e}_{h}^{n-1}-\mathbf{e}_{h}^{n-1} \circ X_{1}^{n}, \bar{D}_{\Delta t} \mathbf{e}_{h}^{n}\right)\right| & \leq \frac{1}{\Delta t}\left\|\mathbf{e}_{h}^{n-1}-\mathbf{e}_{h}^{n-1} \circ X_{1}^{n}\right\|_{0}\left\|\bar{D}_{\Delta t} \mathbf{e}_{h}^{n}\right\|_{0} \leq \alpha_{41}\left\|\mathbf{w}^{n}\right\|_{0, \infty}\left\|\mathbf{e}_{h}^{n-1}\right\|_{1}\left\|\bar{D}_{\Delta t} \mathbf{e}_{h}^{n}\right\|_{0} \\
& \leq \alpha_{41}\left\|\mathbf{w}^{n}\right\|_{0, \infty} \alpha_{1}\left\|\mathrm{D}\left(\mathbf{e}_{h}^{n-1}\right)\right\|_{0}\left\|\bar{D}_{\Delta t} \mathbf{e}_{h}^{n}\right\|_{0} \leq c_{w}\left\|\mathrm{D}\left(\mathbf{e}_{h}^{n-1}\right)\right\|_{0}^{2}+\frac{1}{6}\left\|\bar{D}_{\Delta t} \mathbf{e}_{h}^{n}\right\|_{0}^{2},
\end{aligned}
$$

the equality (46) leads to, for $n=1, \ldots, N_{T}$,

$$
\bar{D}_{\Delta t}\left(\nu\left\|\mathrm{D}\left(\mathbf{e}_{h}^{n}\right)\right\|_{0}^{2}+\frac{\delta_{0}}{2}\left|\epsilon_{h}^{n}\right|_{h}^{2}\right)+\frac{5}{6}\left\|\bar{D}_{\Delta t} \mathbf{e}_{h}^{n}\right\|_{0}^{2} \leq\left\langle\mathbf{r}_{h}^{n}, \bar{D}_{\Delta t} \mathbf{e}_{h}^{n}\right\rangle+c_{w}\left\|\mathrm{D}\left(\mathbf{e}_{h}^{n-1}\right)\right\|_{0}^{2} .
$$

Let $m\left(1 \leq m \leq N_{T}\right)$ be any integer. Summing up (47) for $n=1, \ldots, m$ and using Lemma 7 , we have

$$
\frac{\nu}{2}\left\|\mathrm{D}\left(\mathbf{e}_{h}^{m}\right)\right\|_{0}^{2}+\frac{\delta_{0}}{2}\left|\epsilon_{h}^{m}\right|_{h}^{2}+\frac{\Delta t}{2} \sum_{n=1}^{m}\left\|\bar{D}_{\Delta t} \mathbf{e}_{h}^{n}\right\|_{0}^{2} \leq \frac{c_{w}}{\nu} \Delta t \sum_{n=0}^{m-1} \nu\left\|\mathrm{D}\left(\mathbf{e}_{h}^{n}\right)\right\|_{0}^{2}+c_{\nu, \varepsilon} c_{w, s}\left(\Delta t^{2}+h^{2}\right) .
$$

From Lemma 5 with

$$
x_{n}=\frac{\nu}{2}\left\|\mathrm{D}\left(\mathbf{e}_{h}^{n}\right)\right\|_{0}^{2}+\frac{\delta_{0}}{2}\left|\epsilon_{h}^{n}\right|_{h}^{2}, \quad y_{n}=\frac{1}{2}\left\|\bar{D}_{\Delta t} \mathbf{e}_{h}^{n}\right\|_{0}^{2}, \quad \alpha=\frac{2 c_{w}}{\nu}, \quad \beta=c_{\nu, \varepsilon} c_{w, s}\left(\Delta t^{2}+h^{2}\right),
$$

we have

$$
\left\|\bar{D}_{\Delta t} \mathbf{e}_{h}\right\|_{\ell^{2}\left(L^{2}\right)} \leq c_{\nu, \varepsilon}^{\prime} c_{w, s}^{\prime}(\Delta t+h) .
$$

The first inequality of (12) is obtained by combining the inequality above with the estimate

$$
\begin{aligned}
\left\|\bar{D}_{\Delta t} \mathbf{u}_{h}^{n}-\frac{\partial \mathbf{u}^{n}}{\partial t}\right\|_{0} & \leq\left\|\bar{D}_{\Delta t} \mathbf{e}_{h}^{n}\right\|_{0}+\left\|\bar{D}_{\Delta t} \boldsymbol{\eta}^{n}\right\|_{0}+\left\|\bar{D}_{\Delta t} \mathbf{u}^{n}-\frac{\partial \mathbf{u}^{n}}{\partial t}\right\|_{0} \\
& \leq\left\|\bar{D}_{\Delta t} \mathbf{e}_{h}^{n}\right\|_{0}+\frac{\alpha_{31} h}{\nu \sqrt{\Delta t}}\|(\mathbf{u}, p)\|_{H^{1}\left(t^{n-1}, t^{n} ; H^{2} \times H^{1}\right)}+\sqrt{\frac{\Delta t}{3}}\left\|\frac{\partial^{2} \mathbf{u}}{\partial t^{2}}\right\|_{L^{2}\left(t^{n-1}, t^{n} ; L^{2}\right)}
\end{aligned}
$$

The other inequality of (12) is proved as follows. We have

$$
\begin{aligned}
&\left\|\epsilon_{h}^{n}\right\|_{0} \leq\left\|\left(\mathbf{e}_{h}^{n}, \epsilon_{h}^{n}\right)\right\|_{V \times Q} \leq \frac{1}{\nu \alpha_{30}} \sup _{\left(\mathbf{v}_{h}, q_{h}\right) \in V_{h} \times Q_{h}} \frac{\mathcal{A}_{h}\left(\left(\mathbf{u}_{h}^{n}, \epsilon_{h}^{n}\right),\left(\mathbf{v}_{h}, q_{h}\right)\right)}{\left\|\left(\mathbf{v}_{h}, q_{h}\right)\right\|_{V \times Q}} \\
&= \frac{1}{\nu \alpha_{30}} \sup _{\left(\mathbf{v}_{h}, q_{h}\right) \in V_{h} \times Q_{h}} \frac{\left\langle\mathbf{r}_{h}^{n}, \mathbf{v}_{h}\right\rangle-\frac{1}{\Delta t}\left(\mathbf{e}_{h}^{n}-\mathbf{e}_{h}^{n-1} \circ X_{1}^{n}, \mathbf{v}_{h}\right)}{\left\|\left(\mathbf{v}_{h}, q_{h}\right)\right\|_{V \times Q}} \\
& \leq \frac{1}{\nu \alpha_{30}}\left[\left\|\mathbf{r}_{h 1}^{n}\right\|_{0}+\left\|\mathbf{r}_{h 2}^{n}\right\|_{0}+\left\|\mathbf{r}_{h 3}^{n}\right\|_{-1}+\left\|\mathbf{r}_{h 4}^{n}\right\|_{-1}+\left\|\bar{D}_{\Delta t} \mathbf{e}_{h}^{n}\right\|_{0}+\frac{1}{\Delta t}\left\|\mathbf{e}_{h}^{n-1}-\mathbf{e}_{h}^{n-1} \circ X_{1}^{n}\right\|_{0}\right] \\
& \leq \frac{c_{s}}{\nu \alpha_{30}}\left[\sqrt{\Delta t}\left(\|\mathbf{u}\|_{Z^{2}\left(t^{n-1}, t^{n}\right)}+\|\mathbf{C}\|_{H^{1}\left(t^{n-1}, t^{n} ; L^{2}\right)}\right)+\frac{h}{\nu \sqrt{\Delta t}}\|(\mathbf{u}, p)\|_{H^{1}\left(t^{n-1}, t^{n} ; H^{2} \times H^{1}\right)}\right. \\
&\left.\quad+\left\|\bar{D}_{\Delta t} \mathbf{e}_{h}^{n}\right\|_{0}+\left\|\mathbf{e}_{h}^{n-1}\right\|_{1}+\left\|\mathbf{E}_{h}^{n}\right\|_{0}+\left\|\mathbf{E}_{h}^{n-1}\right\|_{0}+h\right] \quad \text { (by (22a)-(22d)), }
\end{aligned}
$$

which implies the second inequality of (12) from Theorem 1, (49) and the estimate

$$
\left\|p_{h}-p\right\|_{\ell^{2}\left(L^{2}\right)} \leq\left\|\epsilon_{h}\right\|_{\ell^{2}\left(L^{2}\right)}+\left\|\hat{p}_{h}-p\right\|_{\ell^{2}\left(L^{2}\right)} \leq\left\|\epsilon_{h}\right\|_{\ell^{2}\left(L^{2}\right)}+\sqrt{T} \frac{\alpha_{31}}{\nu} h\|(\mathbf{u}, p)\|_{C\left(H^{2} \times H^{1}\right)} .
$$

\section{$6 \quad$ Numerical experiments}

In this section we present numerical results by scheme (6) in order to confirm the theoretical convergence order. We refer to [35] for the detailed description of the algorithm that has been used to perform the numerical simulations. Further numerical experiments for linear scheme (6) as well as for the nonlinear scheme that has been discussed in our previous paper [29], Part I, can also be found in [35]. 
Example. In problem (1) we set $\Omega=(0,1)^{2}$ and $T=0.5$, and we consider three cases for the pair of $\nu$ and $\varepsilon$. Firstly we take both viscosities to be equal $10^{-1}$, i.e., $(\nu, \varepsilon)=\left(10^{-1}, 10^{-1}\right)$. Secondly, we consider the case $(\nu, \varepsilon)=$ $\left(10^{-1}, 10^{-3}\right)$, since the elastic stress viscosity is typically much smaller than the fluid viscosity. Lastly, we set $(\nu, \varepsilon)=(1,0)$. Although the non-diffusive case $\varepsilon=0$ is out of the scope of theoretical analysis of this paper, we dare to carry out the computation to see the performance of scheme (6). The functions $\mathbf{f}, \mathbf{F}, \mathbf{u}^{0}$ and $\mathbf{C}^{0}$ are given such that the exact solution to (1) is as follows:

$$
\begin{aligned}
\mathbf{u}(x, t) & =\left(\frac{\partial \psi}{\partial x_{2}}(x, t),-\frac{\partial \psi}{\partial x_{1}}(x, t)\right), \quad p(x, t)=\sin \left\{\pi\left(x_{1}+2 x_{2}+t\right)\right\}, \\
C_{11}(x, t) & =\frac{1}{2} \sin ^{2}\left(\pi x_{1}\right) \sin ^{2}\left(\pi x_{2}\right) \sin \left\{\pi\left(x_{1}+t\right)\right\}+1, \\
C_{22}(x, t) & =\frac{1}{2} \sin ^{2}\left(\pi x_{1}\right) \sin ^{2}\left(\pi x_{2}\right) \sin \left\{\pi\left(x_{2}+t\right)\right\}+1, \\
C_{12}(x, t) & =\frac{1}{2} \sin ^{2}\left(\pi x_{1}\right) \sin ^{2}\left(\pi x_{2}\right) \sin \left\{\pi\left(x_{1}+x_{2}+t\right)\right\} \quad\left(=C_{21}(x, t)\right), \\
\psi(x, t) & :=\frac{\sqrt{3}}{2 \pi} \sin ^{2}\left(\pi x_{1}\right) \sin ^{2}\left(\pi x_{2}\right) \sin \left\{\pi\left(x_{1}+x_{2}+t\right)\right\} .
\end{aligned}
$$

Proposition 2 and Theorems 1 and 2 hold for any fixed positive constant $\delta_{0}$. Here we simply fix $\delta_{0}=1$. Let $N$ be the division number of each side of the square domain. We set $N=16,32,64,128$ and 256, and (re)define $h:=1 / N$. The time increment is set as $\Delta t=h / 2$. To solve Example we employ scheme (6) with $\left(\mathbf{u}_{h}^{0}, \mathbf{C}_{h}^{0}\right)=\left[\Pi_{h}^{\mathrm{SP}}\left(\mathbf{u}^{0}, 0, \mathbf{C}^{0}\right)\right]_{1,3}$.

For the solution $\left(\mathbf{u}_{h}, p_{h}, \mathbf{C}_{h}\right)$ of scheme (6) and the exact solution $(\mathbf{u}, p, \mathbf{C})$ given by (50) we define the relative errors $\operatorname{Er} i, i=1, \ldots, 6$, by

$$
\begin{array}{lll}
\operatorname{Er} 1=\frac{\left\|\mathbf{u}_{h}-\Pi_{h} \mathbf{u}\right\|_{\ell^{\infty}\left(L^{2}\right)}}{\left\|\Pi_{h} \mathbf{u}\right\|_{\ell^{\infty}\left(L^{2}\right)}}, & \operatorname{Er} 2=\frac{\left\|\mathbf{u}_{h}-\Pi_{h} \mathbf{u}\right\|_{\ell^{2}\left(H^{1}\right)}}{\left\|\Pi_{h} \mathbf{u}\right\|_{\ell^{2}\left(H^{1}\right)}}, & \operatorname{Er} 3=\frac{\left\|p_{h}-\Pi_{h} p\right\|_{\ell^{2}\left(L^{2}\right)}}{\left\|\Pi_{h} p\right\|_{\ell^{2}\left(L^{2}\right)}}, \\
\operatorname{Er} 4=\frac{\left|p_{h}-\Pi_{h} p\right|_{\ell^{2}\left(|\cdot|_{h}\right)}}{\left\|\Pi_{h} p\right\|_{\ell^{2}\left(L^{2}\right)}}, & \operatorname{Er} 5=\frac{\left\|\mathbf{C}_{h}-\Pi_{h} \mathbf{C}\right\|_{\ell^{\infty}\left(L^{2}\right)}}{\left\|\Pi_{h} \mathbf{C}\right\|_{\ell^{\infty}\left(L^{2}\right)}}, & \operatorname{Er} 6=\frac{\left\|\mathbf{C}_{h}-\Pi_{h} \mathbf{C}\right\|_{\ell^{2}\left(H^{1}\right)}}{\left\|\Pi_{h} \mathbf{C}\right\|_{\ell^{2}\left(H^{1}\right)}}
\end{array}
$$

where the same symbol $\Pi_{h}$ has been employed as the scalar and vector versions of the Lagrange interpolation operator.

The values of the errors and the slopes are presented in the tables below, while the corresponding figures show the graphs of the errors versus $h$ in logarithmic scale. Table 1 summarizes the symbols used in the figures. Ta-

\begin{tabular}{|c|c|c|c|c|c|}
\hline \multicolumn{2}{|c|}{$\mathbf{u}_{h}$} & \multicolumn{2}{|c|}{$p_{h}$} & \multicolumn{2}{|c|}{$\mathbf{C}_{h}$} \\
\hline 0 & $\bullet$ & $\triangle$ & $\boldsymbol{\Delta}$ & $\square$ & $\square$ \\
\hline $\operatorname{Er} 1$ & $\operatorname{Er} 2$ & $\operatorname{Er} 3$ & $\operatorname{Er} 4$ & $\operatorname{Er} 5$ & $\operatorname{Er} 6$ \\
\hline
\end{tabular}
bles \& Figures 1, 2 and 3 present the results for the cases $(\nu, \varepsilon)=\left(10^{-1}, 10^{-1}\right),\left(10^{-1}, 10^{-3}\right)$ and $(1,0)$, respectively.

For all the cases it is confirmed that all the errors except $\operatorname{Er} 6$ for $(\nu, \varepsilon)=(1,0)$ are almost of the first order in $h$. These results support Theorems 1 and 2 . Since there is no diffusion for $\mathbf{C}$ in equation (1c) in the case $(\nu, \varepsilon)=(1,0)$, it is natural that the slope of $\operatorname{Er} 6$ does not attain 1 . While the theorems are not proved for $\varepsilon=0$, scheme (6) has worked well in the numerical experiments.

Table 1: Symbols used in the figures.

Remark 9. In the above the difference of $\left(\mathbf{u}_{h}, p_{h}, \mathbf{C}_{h}\right)$ and $\left(\Pi_{h} \mathbf{u}, \Pi_{h} p, \Pi_{h} \mathbf{C}\right)$ are computed. For the difference of $\left(\mathbf{u}_{h}, p_{h}, \mathbf{C}_{h}\right)$ and $(\mathbf{u}, p, \mathbf{C})$ see Appendix A.2.

\section{Conclusions}

In this paper we have presented a linear stabilized Lagrange-Galerkin scheme (6) for the Oseen-type diffusive Peterlin viscoelastic model. The scheme employs the conforming linear finite elements for all unknowns, velocity, pressure and conformation tensor, together with Brezzi-Pitkäranta's stabilization method. In Theorems 1 and 2 we 


\begin{tabular}{rrrrr}
\hline$h$ & $E r 1$ & slope & $E r 2$ & slope \\
\hline $1 / 16$ & $6.29 \times 10^{-2}$ & - & $7.94 \times 10^{-2}$ & - \\
$1 / 32$ & $2.21 \times 10^{-2}$ & 1.51 & $3.14 \times 10^{-2}$ & 1.34 \\
$1 / 64$ & $8.98 \times 10^{-3}$ & 1.30 & $1.32 \times 10^{-2}$ & 1.25 \\
$1 / 128$ & $4.07 \times 10^{-3}$ & 1.14 & $6.35 \times 10^{-3}$ & 1.05 \\
$1 / 256$ & $1.95 \times 10^{-3}$ & 1.07 & $2.86 \times 10^{-3}$ & 1.15 \\
\hline$h$ & $E r 3$ & slope & $E r 4$ & slope \\
\hline $1 / 16$ & $2.02 \times 10^{-1}$ & - & $1.70 \times 10^{-1}$ & - \\
$1 / 32$ & $7.11 \times 10^{-2}$ & 1.50 & $4.99 \times 10^{-2}$ & 1.77 \\
$1 / 64$ & $2.67 \times 10^{-2}$ & 1.41 & $1.86 \times 10^{-2}$ & 1.42 \\
$1 / 128$ & $1.11 \times 10^{-2}$ & 1.27 & $8.39 \times 10^{-3}$ & 1.15 \\
$1 / 256$ & $5.01 \times 10^{-3}$ & 1.15 & $3.69 \times 10^{-3}$ & 1.19 \\
\hline$h$ & $E r 5$ & slope & $E r 6$ & slope \\
\hline $1 / 16$ & $2.80 \times 10^{-2}$ & - & $1.22 \times 10^{-1}$ & - \\
$1 / 32$ & $1.14 \times 10^{-2}$ & 1.30 & $4.41 \times 10^{-2}$ & 1.47 \\
$1 / 64$ & $4.90 \times 10^{-3}$ & 1.21 & $1.72 \times 10^{-2}$ & 1.35 \\
$1 / 128$ & $2.30 \times 10^{-3}$ & 1.09 & $7.64 \times 10^{-3}$ & 1.17 \\
$1 / 256$ & $1.11 \times 10^{-3}$ & 1.05 & $3.59 \times 10^{-3}$ & 1.09 \\
\hline
\end{tabular}

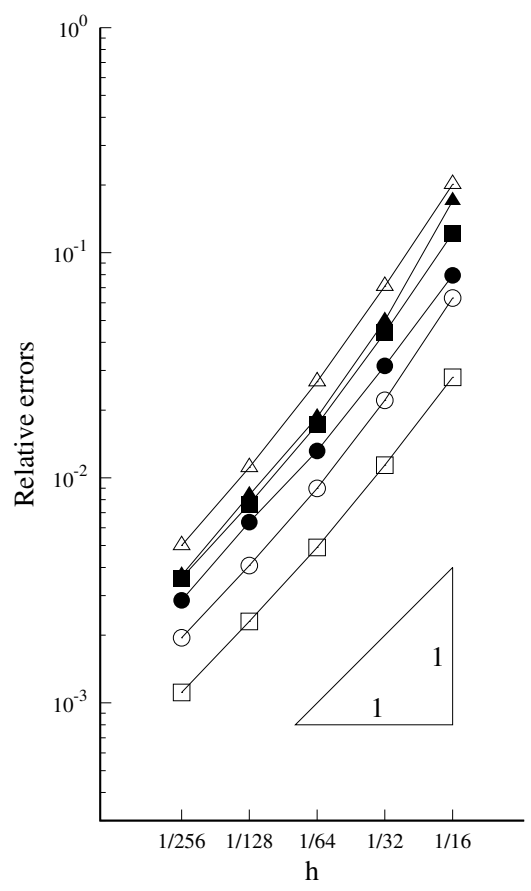

Table \& Figure 1: Errors and slopes for $(\nu, \varepsilon)=\left(10^{-1}, 10^{-1}\right)$.

\begin{tabular}{rrrrr}
\hline$h$ & $E r 1$ & slope & $\operatorname{Er} 2$ & slope \\
\hline $1 / 16$ & $6.14 \times 10^{-2}$ & - & $7.29 \times 10^{-2}$ & - \\
$1 / 32$ & $1.97 \times 10^{-2}$ & 1.64 & $2.91 \times 10^{-2}$ & 1.33 \\
$1 / 64$ & $7.68 \times 10^{-3}$ & 1.36 & $1.21 \times 10^{-2}$ & 1.26 \\
$1 / 128$ & $3.36 \times 10^{-3}$ & 1.19 & $5.93 \times 10^{-3}$ & 1.03 \\
$1 / 256$ & $1.58 \times 10^{-3}$ & 1.09 & $2.66 \times 10^{-3}$ & 1.15 \\
\hline$h$ & $E r 3$ & slope & $E r 4$ & slope \\
\hline $1 / 16$ & $2.50 \times 10^{-1}$ & - & $2.06 \times 10^{-1}$ & - \\
$1 / 32$ & $9.14 \times 10^{-2}$ & 1.45 & $6.08 \times 10^{-2}$ & 1.76 \\
$1 / 64$ & $3.31 \times 10^{-2}$ & 1.46 & $2.11 \times 10^{-2}$ & 1.53 \\
$1 / 128$ & $1.28 \times 10^{-2}$ & 1.37 & $8.78 \times 10^{-3}$ & 1.26 \\
$1 / 256$ & $5.48 \times 10^{-3}$ & 1.23 & $3.74 \times 10^{-3}$ & 1.23 \\
\hline$h$ & $E r 5$ & slope & $E r 6$ & slope \\
\hline $1 / 16$ & $5.01 \times 10^{-2}$ & - & $5.38 \times 10^{-1}$ & - \\
$1 / 32$ & $1.92 \times 10^{-2}$ & 1.38 & $2.54 \times 10^{-1}$ & 1.08 \\
$1 / 64$ & $7.53 \times 10^{-3}$ & 1.35 & $1.05 \times 10^{-1}$ & 1.27 \\
$1 / 128$ & $3.28 \times 10^{-3}$ & 1.20 & $3.88 \times 10^{-2}$ & 1.44 \\
$1 / 256$ & $1.53 \times 10^{-3}$ & 1.10 & $1.35 \times 10^{-2}$ & 1.52 \\
\hline
\end{tabular}

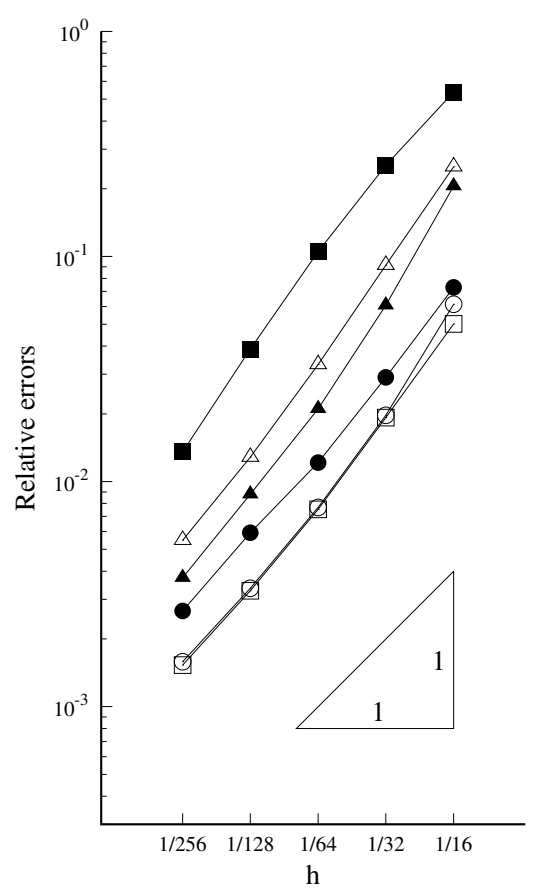

Table \& Figure 2: Errors and slopes for $(\nu, \varepsilon)=\left(10^{-1}, 10^{-3}\right)$. 


\begin{tabular}{rrrrr}
\hline$h$ & $E r 1$ & slope & $E r 2$ & slope \\
\hline $1 / 16$ & $4.51 \times 10^{-2}$ & - & $5.83 \times 10^{-2}$ & - \\
$1 / 32$ & $1.42 \times 10^{-2}$ & 1.67 & $2.36 \times 10^{-2}$ & 1.31 \\
$1 / 64$ & $4.53 \times 10^{-3}$ & 1.65 & $9.85 \times 10^{-3}$ & 1.26 \\
$1 / 128$ & $1.52 \times 10^{-3}$ & 1.58 & $4.89 \times 10^{-3}$ & 1.01 \\
$1 / 256$ & $5.72 \times 10^{-4}$ & 1.41 & $2.10 \times 10^{-3}$ & 1.22 \\
\hline$h$ & $E r 3$ & slope & $E r 4$ & slope \\
\hline $1 / 16$ & $4.78 \times 10^{-1}$ & - & $3.16 \times 10^{-1}$ & - \\
$1 / 32$ & $2.00 \times 10^{-1}$ & 1.26 & $9.18 \times 10^{-2}$ & 1.79 \\
$1 / 64$ & $7.03 \times 10^{-2}$ & 1.51 & $2.95 \times 10^{-2}$ & 1.64 \\
$1 / 128$ & $2.31 \times 10^{-2}$ & 1.60 & $1.17 \times 10^{-2}$ & 1.33 \\
$1 / 256$ & $8.04 \times 10^{-3}$ & 1.52 & $5.01 \times 10^{-3}$ & 1.23 \\
\hline$h$ & $E r 5$ & slope & $E r 6$ & slope \\
\hline $1 / 16$ & $4.93 \times 10^{-2}$ & - & $7.97 \times 10^{-1}$ & - \\
$1 / 32$ & $1.92 \times 10^{-2}$ & 1.36 & $6.05 \times 10^{-1}$ & 0.40 \\
$1 / 64$ & $7.30 \times 10^{-3}$ & 1.39 & $5.32 \times 10^{-1}$ & 0.19 \\
$1 / 128$ & $2.91 \times 10^{-3}$ & 1.33 & $4.04 \times 10^{-1}$ & 0.40 \\
$1 / 256$ & $1.24 \times 10^{-3}$ & 1.22 & $2.74 \times 10^{-1}$ & 0.56 \\
\hline
\end{tabular}

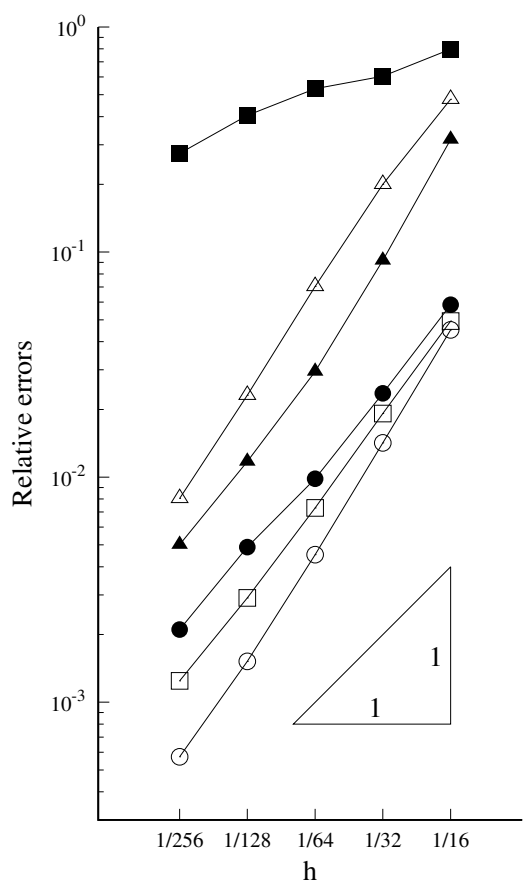

Table \& Figure 3: Errors and slopes for $(\nu, \varepsilon)=(1,0)$.

have established error estimates with the optimal convergence order under mild conditions, $\Delta t=\mathcal{O}(1 / \sqrt{1+|\log h|})$ for $d=2$ and $\Delta t=\mathcal{O}(\sqrt{h})$ for $d=3$. They hold in the standard norms not only for the velocity and the conformation tensor but also for the pressure. The theoretical convergence orders have been confirmed by two-dimensional numerical experiments.

Although we have treated the stabilized scheme to reduce the number of degrees of freedom, the extension of the result to the combination of stable pairs for $(\mathbf{u}, p)$ and conventional elements for $\mathbf{C}$ is straightforwards, e.g., $\mathrm{P} 2 / \mathrm{P} 1 / \mathrm{P} 2$ element. In future we will extend this work to the Peterlin viscoelastic model with the nonlinear convective terms, and compare numerical results with other schemes in some benchmark problems.

We recall that in our previous paper [29], Part I, essentially unconditional stability and error estimates with the optimal convergence order were proved in two space dimensions. There, our analysis allowed to include also the case $\varepsilon=0$.

\section{Acknowledgements}

This research was supported by the German Science Agency (DFG) under the grants IRTG 1529 "Mathematical Fluid Dynamics" and TRR 146 "Multiscale Simulation Methods for Soft Matter Systems", and by the Japan Society for the Promotion of Science (JSPS) under the Japanese-German Graduate Externship "Mathematical Fluid Dynamics". H.M. was partially supported by the German Academic Exchange Service (DAAD). M.L.-M. and H.M. wish to thank B. She (Czech Academy of Science, Prague) for fruitful discussion on the topic. H.N. and M.T. are indebted to JSPS also for Grants-in-Aid for Young Scientists (B), No. 26800091 and for Scientific Research (C), No. 25400212 and Scientific Research (S), No. 24224004, respectively. H.N. is supported by Japan Science and Technology Agency (JST), PRESTO. 


\section{Appendix}

\section{A.1 Proof of Lemma 6}

We prove only $(22 \mathrm{c}),(22 \mathrm{~d}),(22 \mathrm{~h})$ and $(22 \mathrm{l})$, since $(22 \mathrm{a}),(22 \mathrm{~b})$ and $(22 \mathrm{f})$ have been proved in Part I [29] and the other estimates are similarly obtained.

(22c), (22d) and (22h) are obtained as follows:

$$
\begin{aligned}
\left\|\mathbf{r}_{h 3}^{n}\right\|_{-1} & \leq\left\|\left(\operatorname{tr} \mathbf{C}^{n}\right)\left(\mathbf{C}^{n}-\mathbf{C}^{n-1}+\boldsymbol{\Xi}^{n-1}-\mathbf{E}_{h}^{n-1}\right)\right\|_{0} \leq c_{s}\left(\left\|\mathbf{C}^{n}-\mathbf{C}^{n-1}\right\|_{0}+\left\|\boldsymbol{\Xi}^{n-1}\right\|_{0}+\left\|\mathbf{E}_{h}^{n-1}\right\|_{0}\right) \\
& \leq c_{s}\left(\sqrt{\Delta t}\|\mathbf{C}\|_{H^{1}\left(t^{n-1}, t^{n} ; L^{2}\right)}+\alpha_{32} h\left\|\mathbf{C}^{n-1}\right\|_{2}+\left\|\mathbf{E}_{h}^{n-1}\right\|_{0}\right) \\
& \leq c_{s}^{\prime}\left(\left\|\mathbf{E}_{h}^{n-1}\right\|_{0}+\sqrt{\Delta t}\|\mathbf{C}\|_{H^{1}\left(t^{n-1}, t^{n} ; L^{2}\right)}+h\right), \\
\left\|\mathbf{r}_{h 4}^{n}\right\|_{-1} & \leq\left\|\left[\operatorname{tr}\left(\mathbf{\Xi}^{n}-\mathbf{E}_{h}^{n}\right)\right] \mathbf{C}_{h}^{n-1}\right\|_{0} \leq c\left\|\mathbf{C}_{h}^{n-1}\right\|_{0, \infty}\left\|\operatorname{tr}\left(\boldsymbol{\Xi}^{n}-\mathbf{E}_{h}^{n}\right)\right\|_{0} \\
& \leq c^{\prime}\left\|\mathbf{C}_{h}^{n-1}\right\|_{0, \infty}\left(\left\|\mathbf{\Xi}^{n}\right\|_{0}+\left\|\mathbf{E}_{h}^{n}\right\|_{0}\right) \leq c^{\prime}\left\|\mathbf{C}_{h}^{n-1}\right\|_{0, \infty}\left(\alpha_{32} h\left\|\mathbf{C}^{n}\right\|_{2}+\left\|\mathbf{E}_{h}^{n}\right\|_{0}\right) \\
& \leq c_{s}\left\|\mathbf{C}_{h}^{n-1}\right\|_{0, \infty}\left(\left\|\mathbf{E}_{h}^{n}\right\|_{0}+h\right), \\
\left\|\mathbf{R}_{h 4}^{n}\right\|_{0} & =2\left\|\left(\nabla \mathbf{e}_{h}^{n}\right) \mathbf{C}_{h}^{n-1}\right\|_{0} \leq 2 d\left\|\mathbf{C}_{h}^{n-1}\right\|_{0, \infty}\left\|\nabla \mathbf{e}_{h}^{n}\right\|_{0} \leq 2 d\left\|\mathbf{C}_{h}^{n-1}\right\|_{0, \infty}\left\|\mathbf{e}_{h}^{n}\right\|_{1},
\end{aligned}
$$

where in the estimate of $\left\|\mathbf{R}_{h 4}^{n}\right\|_{0}$ the inequality $\|A B\|_{0} \leq d\|A\|_{0, \infty}\|B\|_{0}$ for $A \in L^{\infty}(\Omega)^{d \times d}$ and $B \in L^{2}(\Omega)^{d \times d}$ has been employed.

Finally, (22l) is proved as

$$
\begin{aligned}
\left\|\mathbf{R}_{h 8}^{n}\right\|_{0} & =\left\|\left[\operatorname{tr}\left(\mathbf{C}_{h}^{n-1}+\hat{\mathbf{C}}_{h}^{n-1}\right)\right]\left(\operatorname{tr} \mathbf{E}_{h}^{n-1}\right) \mathbf{C}^{n}\right\|_{0} \leq c_{s}\left(\left\|\mathbf{C}_{h}^{n-1}\right\|_{0, \infty}+\left\|\hat{\mathbf{C}}_{h}^{n-1}\right\|_{0, \infty}\right)\left\|\mathbf{E}_{h}^{n-1}\right\|_{0} \\
& \leq c_{s}^{\prime}\left(\left\|\mathbf{C}_{h}^{n-1}\right\|_{0, \infty}+1\right)\left\|\mathbf{E}_{h}^{n-1}\right\|_{0},
\end{aligned}
$$

where for the last inequality we have used the boundedness of $\left\|\hat{\mathbf{C}}_{h}^{n-1}\right\|_{0, \infty}$ obtained by the estimate

$$
\begin{aligned}
\left\|\hat{\mathbf{C}}_{h}^{n-1}\right\|_{0, \infty} & \leq\left\|\hat{\mathbf{C}}_{h}^{n-1}-\Pi_{h} \mathbf{C}^{n-1}\right\|_{0, \infty}+\left\|\Pi_{h} \mathbf{C}^{n-1}\right\|_{0, \infty} \leq \alpha_{21} D(h)\left\|\hat{\mathbf{C}}_{h}^{n-1}-\Pi_{h} \mathbf{C}^{n-1}\right\|_{1}+\|\mathbf{C}\|_{C\left(L^{\infty}\right)} \\
& \leq \alpha_{21} D(h)\left(\left\|\hat{\mathbf{C}}_{h}^{n-1}-\mathbf{C}^{n-1}\right\|_{1}+\left\|\mathbf{C}^{n-1}-\Pi_{h} \mathbf{C}^{n-1}\right\|_{1}\right)+\|\mathbf{C}\|_{C\left(L^{\infty}\right)} \\
& \leq \alpha_{21} D(h)\left(\alpha_{32} h\left\|\mathbf{C}^{n-1}\right\|_{2}+\alpha_{20} h\left\|\mathbf{C}^{n-1}\right\|_{2}\right)+\|\mathbf{C}\|_{C\left(L^{\infty}\right)} \\
& \leq \alpha_{21} h D(h)\left(\alpha_{20}+\alpha_{32}\right)\|\mathbf{C}\|_{C\left(H^{2}\right)}+\|\mathbf{C}\|_{C\left(L^{\infty}\right)} \\
& \leq \alpha_{21} h_{1} D\left(h_{1}\right)\left(\alpha_{20}+\alpha_{32}\right)\|\mathbf{C}\|_{C\left(H^{2}\right)}+\|\mathbf{C}\|_{C\left(L^{\infty}\right)} \leq c_{s} .
\end{aligned}
$$

\section{A.2 Difference of $\left(\mathbf{u}_{h}, p_{h}, \mathbf{C}_{h}\right)$ and $(\mathbf{u}, p, \mathbf{C})$ in Example.}

In Section 6 we have computed the difference of $\left(\mathbf{u}_{h}, p_{h}, \mathbf{C}_{h}\right)$ and $\left(\Pi_{h} \mathbf{u}, \Pi_{h} p, \Pi_{h} \mathbf{C}\right)$. Here, we give additional information on the error between $\left(\mathbf{u}_{h}, p_{h}, \mathbf{C}_{h}\right)$ and $(\mathbf{u}, p, \mathbf{C})$. We introduce a numerical integration formula of degree five with seven quadrature points for each triangle, and we denote the norm derived by the formula by adding the prime to the corresponding norm,

$$
\|\psi\|_{L^{2}(\Omega)^{\prime}}:=\left\{\sum_{K \in \mathcal{T}_{h}} \operatorname{meas}(K) \sum_{i=1}^{7}\left|\psi\left(a_{i}^{K}\right)\right|^{2} w_{i}\right\}^{1 / 2} \approx\|\psi\|_{L^{2}(\Omega)},
$$

where $\left\{\left(a_{i}^{K}, w_{i}\right)\right\}$ is a set of pairs of quadrature point and weight on $K \in \mathcal{T}_{h}$. When $\psi$ is a function in P1 finite element space, it holds that $\|\psi\|_{L^{2}(\Omega)^{\prime}}=\|\psi\|_{L^{2}(\Omega)}$. We abbreviate $\|\psi\|_{L^{2}(\Omega)^{\prime}}$ as $\|\psi\|_{L^{2 \prime}}$. In the following the symbol I means that the numerical integration is used in place of the exact integration. We define the relative errors $E r k^{\prime}$, $k=1, \ldots, 6$, by

$$
\begin{array}{lll}
\operatorname{Er} 1^{\prime}:=\frac{\left\|\mathbf{u}_{h}-\mathbf{u}\right\|_{\ell^{\infty}\left(L^{2 \prime}\right)}}{\left\|\Pi_{h} \mathbf{u}\right\|_{\ell^{\infty}\left(L^{2}\right)}}, & \operatorname{Er} 2^{\prime}:=\frac{\left\|\mathbf{u}_{h}-\mathbf{u}\right\|_{\ell^{2}\left(H^{1 \prime}\right)}}{\left\|\Pi_{h} \mathbf{u}\right\|_{\ell^{2}\left(H^{1}\right)}}, & \operatorname{Er} 3^{\prime}:=\frac{\left\|p_{h}-p\right\|_{\ell^{2}\left(L^{2 \prime}\right)}}{\left\|\Pi_{h} p\right\|_{\ell^{2}\left(L^{2}\right)}}, \\
\operatorname{Er~} 4^{\prime}:=\frac{\left|p_{h}-p\right|_{\ell^{2}\left(|\cdot|_{h}^{\prime}\right)}}{\left\|\Pi_{h} p\right\|_{\ell^{2}\left(L^{2}\right)}}, & \operatorname{Er} 5^{\prime}:=\frac{\left\|\mathbf{C}_{h}-\mathbf{C}\right\|_{\ell^{\infty}\left(L^{2 \prime}\right)}}{\left\|\Pi_{h} \mathbf{C}\right\|_{\ell^{\infty}\left(L^{2}\right)}}, & \operatorname{Er} 6^{\prime}:=\frac{\left\|\mathbf{C}_{h}-\mathbf{C}\right\|_{\ell^{2}\left(H^{1 \prime}\right)}}{\left\|\Pi_{h} \mathbf{C}\right\|_{\ell^{2}\left(H^{1}\right)}} .
\end{array}
$$


We deal with the case $(\nu, \varepsilon)=\left(10^{-1}, 10^{-1}\right)$. Table 2 shows the comparison of the values of $E r k^{\prime}$ with those of $\operatorname{Er} k$, which reflects that convergence orders of $E r k$ are almost same with those of $E r k^{\prime}$, though the values of $\operatorname{Er} 2^{\prime}$ are about three to four times larger than $\operatorname{Er} 2$. Therefore, the computation of the difference of $\left(\mathbf{u}_{h}, p_{h}, \mathbf{C}_{h}\right)$ and $\left(\Pi_{h} \mathbf{u}, \Pi_{h} p, \Pi_{h} \mathbf{C}\right)$ is sufficient in order to observe the behavior of convergence of $\left(\mathbf{u}_{h}, p_{h}, \mathbf{C}_{h}\right)$ to $(\mathbf{u}, p, \mathbf{C})$.

Table 2: Comparison of $\operatorname{Er} k$ with $\operatorname{Er} k^{\prime}, k=1, \ldots, 6$, for $(\nu, \varepsilon)=\left(10^{-1}, 10^{-1}\right)$.

\begin{tabular}{|c|c|c|c|c|c|c|c|c|}
\hline$h$ & $\operatorname{Er} 1$ & slope & $\operatorname{Er} 1^{\prime}$ & slope & $\operatorname{Er} 2$ & slope & $\operatorname{Er} 2^{\prime}$ & slope \\
\hline $1 / 16$ & $6.29 \times 10^{-2}$ & - & $8.15 \times 10^{-2}$ & - & $7.94 \times 10^{-2}$ & - & $1.94 \times 10^{-1}$ & - \\
\hline $1 / 32$ & $2.21 \times 10^{-2}$ & 1.51 & $2.68 \times 10^{-2}$ & 1.60 & $3.14 \times 10^{-2}$ & 1.34 & $9.20 \times 10^{-2}$ & 1.08 \\
\hline $1 / 64$ & $8.98 \times 10^{-3}$ & 1.30 & $1.02 \times 10^{-2}$ & 1.39 & $1.32 \times 10^{-2}$ & 1.25 & $4.54 \times 10^{-2}$ & 1.02 \\
\hline $1 / 128$ & $4.07 \times 10^{-3}$ & 1.14 & $4.40 \times 10^{-3}$ & 1.22 & $6.35 \times 10^{-3}$ & 1.05 & $2.27 \times 10^{-2}$ & 1.00 \\
\hline $1 / 256$ & $1.95 \times 10^{-3}$ & 1.07 & $2.03 \times 10^{-3}$ & 1.12 & $2.86 \times 10^{-3}$ & 1.15 & $1.12 \times 10^{-2}$ & 1.02 \\
\hline$h$ & $\operatorname{Er} 3$ & slope & $\operatorname{Er} 3^{\prime}$ & slope & $\operatorname{Er} 4$ & slope & $\operatorname{Er} 4^{\prime}$ & slope \\
\hline $1 / 16$ & $2.02 \times 10^{-1}$ & - & $2.13 \times 10^{-1}$ & - & $1.70 \times 10^{-1}$ & - & $1.81 \times 10^{-1}$ & - \\
\hline $1 / 32$ & $7.11 \times 10^{-2}$ & 1.50 & $7.38 \times 10^{-2}$ & 1.53 & $4.99 \times 10^{-2}$ & 1.77 & $5.21 \times 10^{-2}$ & 1.80 \\
\hline $1 / 64$ & $2.67 \times 10^{-2}$ & 1.41 & $2.73 \times 10^{-2}$ & 1.43 & $1.86 \times 10^{-2}$ & 1.42 & $1.90 \times 10^{-2}$ & 1.46 \\
\hline $1 / 128$ & $1.11 \times 10^{-2}$ & 1.27 & $1.12 \times 10^{-2}$ & 1.28 & $8.39 \times 10^{-3}$ & 1.15 & $8.44 \times 10^{-3}$ & 1.17 \\
\hline $1 / 256$ & $5.01 \times 10^{-3}$ & 1.15 & $5.03 \times 10^{-3}$ & 1.16 & $3.69 \times 10^{-3}$ & 1.19 & $3.69 \times 10^{-3}$ & 1.19 \\
\hline$h$ & $\operatorname{Er} 5$ & slope & $\operatorname{Er} 5^{\prime}$ & slope & $\operatorname{Er} 6$ & slope & $\operatorname{Er} 6^{\prime}$ & slope \\
\hline $1 / 16$ & $2.80 \times 10^{-2}$ & - & $2.80 \times 10^{-2}$ & - & $1.22 \times 10^{-1}$ & - & $1.64 \times 10^{-1}$ & 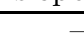 \\
\hline $1 / 32$ & $1.14 \times 10^{-2}$ & 1.30 & $1.14 \times 10^{-2}$ & 1.30 & $4.41 \times 10^{-2}$ & 1.47 & $6.95 \times 10^{-2}$ & 1.24 \\
\hline $1 / 64$ & $4.90 \times 10^{-3}$ & 1.21 & $4.90 \times 10^{-3}$ & 1.21 & $1.72 \times 10^{-2}$ & 1.35 & $3.22 \times 10^{-2}$ & 1.11 \\
\hline $1 / 128$ & $2.30 \times 10^{-3}$ & 1.09 & $2.30 \times 10^{-3}$ & 1.09 & $7.64 \times 10^{-3}$ & 1.17 & $1.56 \times 10^{-2}$ & 1.04 \\
\hline $1 / 256$ & $1.11 \times 10^{-3}$ & 1.05 & $1.11 \times 10^{-3}$ & 1.05 & $3.59 \times 10^{-3}$ & 1.09 & $7.69 \times 10^{-3}$ & 1.02 \\
\hline
\end{tabular}

\section{References}

[1] M. Aboubacar, H. Matallah, and M.F. Webster. Highly elastic solutions for Oldroyd-B and Phan-Thien/Tanner fluids with a finite volume/element method: planar contraction flows. Journal of Non-Newtonian Fluid Mechanics, 103:65-103, 2002.

[2] M.A. Alves, P.J. Oliveira, and F.T. Pinho. Benchmark solutions for the flow of Oldroyd-B and PTT fluids in planar contractions. Journal Non-Newtonian Fluid Mechanics, 110:45-75, 2003.

[3] J. Baranger and D. Sandri. Finite element approximation of viscoelastic fluid flow. Numerische Mathematik, 63:13-27, 1992.

[4] J.W. Barrett and E. Süli. Existence and equilibration of global weak solutions to kinetic models for dilute polymers II: Hookean-type models. Mathematical Models and Methods in Applied Sciences, 22, 1150024, 2012.

[5] A.V. Bhave, R.C. Armstrong, and R.A. Brown. Kinetic theory and rheology of dilute, nonhomogeneous polymer solutions. Journal of Chemical Physics, 95:2988-3000, 1991.

[6] A. Bonito, P. Clément, and M. Picasso. Mathematical and numerical analysis of a simplified time-dependent viscoelastic flow. Numerische Mathematik, 107:213-255, 2007.

[7] A. Bonito, M. Picasso, and M. Laso. Numerical simulation of 3D viscoelastic flows with free surfaces. Journal of Computational Physics, 215:691-716, 2006.

[8] J. Bonvin, M. Picasso, and R. Stenberg. GLS and EVSS methods for a three-field Stokes porblem arising from viscoelastic flows. Computer Methods in Applied Mechanics and Engineering, 190:3893-3941, 2001.

[9] K. Boukir, Y. Maday, B. Métivet, and E. Razafindrakoto. A high-order characteristics/finite element method for the incompressible Navier-Stokes equations. International Journal for Numerical Methods in Fluids, 25:1421-1454, 1997. 
[10] S. Boyaval, T. Lelièvre, and C. Mangoubi. Free-energy-dissipative schemes for the Oldroyd-B model. ESAIM: M2AN, 43:523-561, 2009.

[11] S.C. Brenner and L.R. Scott. The Mathematical Theory of Finite Element Methods. Springer, New York, 3rd edition, 2008.

[12] F. Brezzi and J. Douglas Jr. Stabilized mixed methods for the Stokes problem. Numerische Mathematik, 53:225-235, 1988.

[13] F. Brezzi and J. Pitkäranta. On the stabilization of finite element approximations of the Stokes equations. In W. Hackbusch, editor, Efficient Solutions of Elliptic Systems, pages 11-19, Wiesbaden, 1984. Vieweg.

[14] P.G. Ciarlet. The Finite Element Method for Elliptic Problems. North-Holland, Amsterdam, 1978.

[15] M.J. Crochet and R. Keunings. Finite element analysis of die swell of a highly elastic fluid. Journal of Non-Newtonian Fluid Mechanics, 10:339-356, 1982.

[16] J. de Frutos and B. García-Archilla. Grad-div stabilization for the evolutionary Oseen problem with inf-sup stable finite elements. Journal of Scientific Computing, 66:991-1024, 2016.

[17] P. Degond and H. Liu. Kinetic models for polymers with inertial effects. Network and Heterogeneous Media, 4:625-647, 2009.

[18] Y. Fan. A comparative study of the discontinuous Galerkin and continuous SUPG finite element methods for computation of viscoelastic flows. Computational Methods in Applied Mechanics and Engineering, 141:47-65, 1997.

[19] Y. Fan, R.I. Tanner and N. Phan-Thien. Galerkin/least-square finite-element methods for steady viscoelastic flows. Journal of Non-Newtonian Fluid Mechanics, 84:233-256, 1999.

[20] R. Fattal and R. Kupferman. Constitutive laws for the matrix-logarithm of the conformation tensor. Journal of Non-Newtonian Fluid Mechanics, 123:281-285, 2004.

[21] R. Fattal and R. Kupferman. Time-dependent simulation of viscoelastic flows at high Weissenberg number using the log-conformation representation. Journal of Non-Newtonian Fluid Mechanics, 126:23-37, 2005.

[22] M. Fortin and A. Fortin. A new approach for the FEM simulations of viscoelastic flows. Journal of NonNewtonian Fluid Mechanics, 32:295-310, 1989.

[23] L.P. Franca and R. Stenberg. Error analysis of some Galerkin least squares methods for the elasticity equations. SIAM Journal on Numerical Analysis, 28:1680-1697, 1991.

[24] R. Guénette and M. Fortin. A new mixed finite element method for computing viscoelastic flows. Journal of Non-Newtonian Fluid Mechanics, 60:27-52, 1995.

[25] J.G. Heywood and R. Rannacher. Finite-element approximation of the nonstationary Navier-Stokes problem. Part IV: error analysis for second-order time discretization. SIAM Journal on Numerical Analysis, 27(2):353384, 1990.

[26] R. Keunings. On the high Weissenberg number problem. Journal of Non-Newtonian Fluid Mechanics, 20:209226, 1986.

[27] Y.-J. Lee and J. Xu. New formulations, positivity preserving discretizations and stability analysis for nonNewtonian flow models. Computer Methods in Applied Mechanics and Engineering, 195:1180-1206, 2006.

[28] Y.-J. Lee, J. Xu, and C.-S. Zhang. Global existence, uniqueness and optimal solvers of discretized viscoelastic flow models. Mathematical Models and Methods in Applied Sciences, 21(8):1713-1732, 2011.

[29] M. Lukáčová-Medvid'ová, H. Mizerová, H. Notsu, and M. Tabata. Numerical analysis of the Oseen-type Peterlin viscoelastic model by the stabilized Lagrange-Galerkin method, Part I: A nonlinear scheme. ESAIM: M2AN, in press. DOI: 10.1051/m2an/2016078.

[30] M. Lukáčová-Medvid'ová, H. Mizerová, and Š. Nečasová. Global existence and uniqueness result for the diffusive Peterlin viscoelastic model. Nonlinear Analysis: Theory, Methods \& Applications, 120:154-170, 2015. 
[31] M. Lukáčová-Medvid'ová, H. Mizerová, Š. Nečasová, and M. Renardy. Global existence result for the generalized Peterlin viscoelastic model. Submitted to SIAM Journal of Mathematical Analysis, 2016.

[32] M. Lukáčová-Medvid'ová, H. Notsu, and B. She. Energy dissipative characteristic schemes for the diffusive Oldroyd-B viscoelastic fluid. International Journal for Numerical Methods in Fluids, 81:523-557, 2016. DOI: $10.1002 /$ fld.4195.

[33] J. Nečas. Les Méthods Directes en Théories des Équations Elliptiques. Masson, Paris, 1967.

[34] J.M. Marchal and M.J. Crochet. A new mixed finite element for calculating viscoelastic flow. Journal of Non-Newtonian Fluid Mechanics, 26:77-114, 1987.

[35] H. Mizerová. Analysis and numerical solution of the Peterlin viscoelastic model. 2015. PhD thesis, University of Mainz, Germany.

[36] L. Nadau and A. Sequeira. Numerical simulations of shear-dependent viscoelastic flows with a combined finite element-finite volume method. Computers $\&$ Mathematics with Applications, 53:547-568, 2007.

[37] H. Notsu and M. Tabata. Error estimates of stable and stabilized Lagrange-Galerkin schemes for natural convection problems. arXiv:1511.01234 [math.NA].

[38] H. Notsu and M. Tabata. Error estimates of a pressure-stabilized characteristics finite element scheme for the Oseen equations. Journal of Scientific Computing, 65(3):940-955, 2015.

[39] H. Notsu and M. Tabata. Error estimates of a stabilized Lagrange-Galerkin scheme for the Navier-Stokes equations. ESAIM: M2AN, 50(2):361-380, 2016.

[40] M.A. Olshanskii and A. Reusken. Grad-div stabilization for Stokes equations. Mathematics of Computation, 73(248):1699-1718, 2003.

[41] R.G. Owens and T.N. Philips. Computational Rheology. Imperial College Press, 2002.

[42] R.G. Owens, C. Chauvière, and T.N. Philips. A locally-upwinded spectral technique (LUST) for viscoelastic flows. Journal of Non-Newtonian Fluid Mechanics, 108:49-71, 2002.

[43] A. Peterlin. Hydrodynamics of macromolecules in a velocity field with longitudinal gradient. Journal of Polymer Science Part B: Polymer Letters, 4:287-291, 1966.

[44] M. Picasso and J. Rappaz. Existence, a priori and a posteriori error estimates for a nonlinear three-field problem arising from Oldroyd-B viscoelastic flows. ESAIM: M2AN, 35:879-897, 2001.

[45] D. Rajagopalan, R.C. Armstrong and R.A. Brown. Finite element methods for calculation of steady, viscoelastic flow using constitutive equations with a Newtonian viscosity. Journal of Non-Newtonian Fluid Mechanics, $36: 159-192,1990$.

[46] M. Renardy. Mathematical Analysis of Viscoelastic Flows. CBMS-NSF Conference Series in Applied Mathematics 73. SIAM, New York, 2000.

[47] M. Renardy. Mathematical analysis of viscoelastic fluids. In Handbook of Differential Equations: Evolutionary Equations, volume 4, pages 229-265, Amsterdam, 2008. North-Holland.

[48] M. Renardy. The mathematics of myth: Yield stress behaviour as a limit of non-monotone constitutive theories. Journal of Non-Newtonian Fluid Mechanics, 165:519-526, 2010.

[49] M. Renardy and T. Wang. Large amplitude oscillatory shear flows for a model of a thixotropic yield stress fluid. Journal of Non-Newtonian Fluid Mechanics, 222:1-17, 2015.

[50] H. Rui and M. Tabata. A second order characteristic finite element scheme for convection-diffusion problems. Numerische Mathematik, 92:161-177, 2002.

[51] E. Süli. Convergence and nonlinear stability of the Lagrange-Galerkin method for the Navier-Stokes equations. Numerische Mathematik, 53:459-483, 1988.

[52] M. Tabata and S. Uchiumi. An exactly computable Lagrange-Galerkin scheme for the Navier-Stokes equations and its error estimates. Mathematics of Computation, in press. DOI: 10.1090/mcom/3222. 
[53] Wang K. A new discrete EVSS method for the viscoelastic flows. Computers and Mathematics with Applications, 65:609-615, 2013.

[54] P. Wapperom, R. Keunings, and V. Legat. The backward-tracking Lagrangian particle method for transient viscoelastic flows. Journal of Non-Newtonian Fluid Mechanics, 91:273-295, 2000. 